\title{
Immunoglobulin kappa light chain produced by cardiomyocytes and participates in maintaining intercalated disc integrity
}

Xiaoyan Qiu ( $\nabla$ qiuxy@bjmu.edu.cn )

Department of Immunology, School of Basic Medical Sciences, Peking University

Zhu Zhu

Peking University

Zhan Shi

Peking University

Meng Zhang

Aerospace Center Hospital, Beijing

Wenjing Zhou

Peking University

\section{Feng Lan}

Zhejiang University https://orcid.org/0000-0002-9038-4014

Qinyuan Liao

Guilin Medicial University

\section{Article}

Keywords: Arrhythmogenic cardiomyopathy, Immunoglobin(Ig), Intercalated disc(ICD), Myocardial contraction, Plectin

Posted Date: December 2nd, 2020

DOI: https://doi.org/10.21203/rs.3.rs-114990/v1

License: (c) (1) This work is licensed under a Creative Commons Attribution 4.0 International License. Read Full License 


\section{Abstract}

It is widely accepted that immunoglobulins (Igs) are produced only by B cells and function as antibodies. However, growing evidence has proven that almost all non-B cells also produce Igs with nonconventional roles, such as promotion of cell survival, proliferation and migration. In this study, we identified Ig light chain ( $\mathrm{K}$ chain) expression in mouse and human cardiomyocytes, especially on intercalated discs (ICDs). Unexpectedly, conditional knockout (cKO) of Igk in adult cardiomyocytes in mice resulted in significant hypotension, a rapid decrease in cardiac contractility and conduction defects. Histologically, lgk knockout in mouse cardiomyocytes led to structural disruption of intercalated discs (ICDs) and loss of localization of adhesion-related N-cadherin and CX43 on ICDs. Mechanistic investigation indicated that Igk can bind with plectin/desmoplakin, a complex that connects desmin and desmosomes and enhances the protein stability of plectin. In conclusion, Our findings identify Igk expressed by cardiomyocytes as a new ICDrelated molecule that participates in cardiomyocyte contraction and conduction by stabilizing plectin.

\section{Introduction}

Immunoglobulins (Igs) are members of a classical immune molecule family and mainly exert antibody function. For several decades, B cells and differentiated plasma cells were thought to be the only sources of Igs. However, in recent years, many studies from our group and others have revealed that Igs are not only produced by B cells but also expressed by non-B cell lineages, including epithelial cells, neurons and germ cells of the testis ${ }^{1-8}$. In addition to serving as natural antibodies for immune defense, non-B Igs also exhibit diverse biological functions; for example, renal podocyte-derived IgG promotes cell adhesion and survival ${ }^{9,10}$. Cancer-derived IgGs are involved in the survival, adhesion, migration and metastasis of cancer cells by forming focal adhesions via binding to integrin ${ }^{11-13}$. These findings suggest that Igs are widely produced by many kinds of lineages other than B cells and that their physiological or pathological functions are diverse.

The heart is generally considered a dynamic organ and has the main function of maintaining blood circulation. So far, there is no evidence revealing that heart function is related to Igs. However, a number of clinical phenomena have shown that increased Igs are closely related to heart disease; for instance, IgE rapidly increases in the blood in acute myocardial infarction ${ }^{14,15}$, local IgM deposition occurs in injured cardiomyocytes 16,17 , and free Ig light chain (FLC) is frequently increased in the serum of patients with heart failure (HF) and has been suggested to be a novel marker of the prognosis of heart failure ${ }^{18}$. In particular, deposition of LC in heart tissue, which affects the mechanical and structural properties of heart tissue and exhibits direct toxicity to cardiomyocytes, is the pathogenic marker of incurable myocardial amyloidosis ${ }^{19-22}$. Although the significance of Igs in heart disease has been thoroughly studied, the origin of these Igs is currently thought to be restricted to B cells, which limits the understanding of the function of these Igs. Given that our studies and those by other groups have shown that Igs can be widely produced by non-B cells, we wondered whether cardiomyocytes can also produce Igs. In 2009, Mehta et al found Ig transcripts of both heavy chain and light chain in mouse heart tissue and HL-1 cells (mouse 
myocardial cells) ${ }^{23}$. In 2017, we reported that IgM heavy chain exists in cardiomyocytes from both WT and $B$ cell-deficient $(\mu \mathrm{MT})$ mice and that ischaemia and hypoxia promote the expression and secretion of $\operatorname{lgM}{ }^{24}$. These studies demonstrated that Igs can be produced by cardiomyocytes, and the physiological function of cardiomyocyte-derived Igs (CM-lgs) needs to be determined.

Classical Ig consists of two identical heavy chains and two identical light chains. There are five types of heavy chains, including $\lg \mathrm{M}, \lg \mathrm{G}, \lg \mathrm{A}, \lg \mathrm{D}$ and $\lg \mathrm{E}$. There are two types of light chains, namely, $\mathrm{k}$ and $\mathrm{I}$. Each of the light chains can form a complete Ig molecule with any of the heavy chains mentioned above, but the frequency of the two light chains in mice is different, and their ratio is 20:1. In fact, in our previous work, we found that $\lg M$ as well as $\lg G, \lg A$ and $\lg E$ are expressed in cardiomyocytes. However, the main Ig light chain in cardiomyocytes is Igk. Therefore, in this study, we focused on demonstrating Igk expression by cardiomyocytes and identifying the function of lgk. We found that lgk acts as a novel cardiac ICD-related protein to maintain the structural integrity of ICDs and the cardiac functions of the adult heart.

\section{Experimental Procedures}

\section{Animal and human heart specimen}

Adult Balb/c mice and C57BL/6 mice (8 weeks) were obtained from Vital River (Charles River Laboratories) and $\mu \mathrm{MT}$ mice (Balb/c background) were a gift from Professor Zhihai Qin (Institute of Biophysics, Chinese Academy of Sciences). Mice were perfused with PBS for 5 minutes, and the heart was removed. Conditions of animal housing and all experimental procedures were conducted under institutional guidelines provided by the Institutional Animal Care and Use Committee of China.

human heart autopsy specimen was a gift from Qingyuan Liao (Guilin Medical University) and human iPS-CM was a gift from Feng Lan (Anzhen hospital).

\section{HL-1 myocardial cell culture}

HL-1, an adult mouse atrial myocardial cell line, was a gift from Dr. W.C.Claycomb (Louisiana State University, New Orleans, LA) ${ }^{25}$. HL-1 cells were cultured in Claycomb medium (Sigma-Aldrich) supplemented with $10 \%$ FBS (HyClone), $100 \mathrm{U} / \mathrm{ml}$ penicillin, $100 \mu \mathrm{g} / \mathrm{ml}$ streptomycin, $2 \mathrm{mM}$ L-glutamine, and $0.1 \mathrm{mM}$ norepinephrine (Sigma-Aldrich) at $37^{\circ} \mathrm{C}$ under $5 \% \mathrm{CO} 2$ in $0.02 \%$ gelatin coated flasks containing $0.5 \%$ fibronectin (Sigma-Aldrich).

\section{Isolation and culture of neonatal mouse ventricular myocytes}

Ventricular cardiomyocytes were isolated from the left ventricles of neonatal mice using enzymatic digestions containing $0.08 \%$ trypsin (Gibco BRL) and $0.1 \%$ collagenase (Sigma-Aldrich) for 8 min at 37 ${ }^{\circ} \mathrm{C}$. Isolated cardiomyocytes were re-suspended in Dulbecco's modified Eagle's medium (DMEM) containing $10 \%$ fetal bovine serum (FBS) and $1 \%$ penicillin-streptomycin and then plated at a density of 
$6 \times 10^{5} / \mathrm{dish}$ and cultured for $1.5 \mathrm{~h}$. The supernatant was subsequently collected and centrifuged at $500 \mathrm{~g}$ for $10 \mathrm{~min}$ and resuspended in $5 \mathrm{ml}$ culture medium containing $100 \mathrm{mmol} / \mathrm{L} 5{ }^{\prime} \mathrm{BrdU}$ (Sigma-Aldrich) and plated in $35 \mathrm{~mm}$ culture dishes for $48 \mathrm{~h}$.

\section{Isolation of adult mouse ventricular myocytes and single cardiomyocyte dissociation.}

Ventricular myocytes were isolated from the Langendorff-perfused hearts of adult C57BL/6 mice. Briefly, the mice were anaesthetized with pentobarbital and the heart was removed and mounted on the Langendorff apparatus and perfused with $\mathrm{Ca}^{2+}$-free Tyrode's solution to eliminate the blood. Then the heart was perfused with Tyrode's solution containing $0.67 \mathrm{mg} / \mathrm{ml}$ of collagenase type II (Worthington) for $30 \mathrm{~min}$ for digestion. When the heart became flaccid, remove the ventricle and cut into small pieces and then pelleted by centrifugation at 500rpm for $1 \mathrm{~min}$ at room temperature and resuspended in Tyrode's solution. The calcium concentration was then gradually increased to $500 \mathrm{mM}$ over $80 \mathrm{~min}$. The supernatant was discarded and the pellet was resuspended in PBS containing $1 \mathrm{mg} / \mathrm{ml} \mathrm{BSA}$. Cardiomyocyte single cells were manually picked under the microscope by mouth pipette. To make sure that only single cells were collected, the solution was visually inspected under the microscope and was discarded if multiple cells were observed. Volume of liquid was kept below $0.5 \mu$ l. Cells were then transferred to a $0.2 \mathrm{ml}$ thin-wall PCR tube containing lysate buffer.

\section{RNA extraction and cDNA synthesis}

Myocardial single cell RNA extraction and cDNA synthesis were carried out according to Tang's methods as described ${ }^{26,27}$. In briefly, myocardial single cells were picked by capillary pipette and reverse transcription was then performed directly on the whole cell lysate. After this, the free primers were removed by ExoSAP-IT and a poly(A) tail was added to the $3^{\prime}$ end of the first-strand cDNA by Terminal Deoxynucleotidyl Transferase. Then the single cell cDNAs were amplified following PCR program: $95^{\circ} \mathrm{C}$ for $3 \mathrm{~min}$, then $27 \mathrm{cycles}$ of $95^{\circ} \mathrm{C}$ for $30 \mathrm{sec}, 67^{\circ} \mathrm{C}$ for $1 \mathrm{~min}$, and $72{ }^{\circ} \mathrm{C}$ for $6 \mathrm{~min}$ ( $6 \mathrm{sec}$ each cycle). After this step, all cDNAs have been amplified. The single cell cDNA PCR product can be saved at $-80{ }^{\circ} \mathrm{C}$ for 2 months.

Total RNA was extracted from frozen heart tissue as well as HL-1 cells by using Trizol Reagent (Invitrogen). Reverse transcription (RT) was carried out with the First Stand cDNA Synthesis Kit (Thermo Scientific) according to the manufacturer's protocol.

\section{PCR amplification and DNA sequencing}

After the cDNA was obtained, specific primers were used to amplify the lgk, CD20, Myh6, Myh7, cTnT, CTnl and GAPDH. The sequences of all PCR primers are listed in Supplementary Table 1. Touchdown PCR was performed for amplification. PCR products were cloned into a pGEM-T Easy Vector (Promega). These clones were analyzed by the Sanger method using an ABI 3100 Genetic Analyzer (Applied Biosystems). The sequences of VkJk were compared with those in the BLAST and Immunogenetics databases to 
identify the best matches for germline gene segments and VJ junctions. All VkJk sequences were shown in supplementary table 2 .

The DNA concentration of eluted PCR products were measured and $146 \mathrm{ng}$ of DNA was pooled for sequencing. The following sequencing using the $2 \times 250$ bp Illumina MiSeq platform were performed by Novogene Corporation (Beijing, China). Single cell of WT mice $(n=17)$ and MMT mice $(n=3)$ was analyzed. The usage of Vk was shown in supplementary table 3.

\section{RNA interference}

The sequence for Igk siRNA was as follows: siRNA-1: GAAGATTGATGGCAGTGAA. siRNA-2: GAACGACATAACAGCTATA. These were used to transfect HL-1 cells with the Lipofectamine 3000 kit (Invitrogen) according to the manual; a mock group was transfected with control siRNA. Cells were harvested $24 \mathrm{~h}$ and $48 \mathrm{~h}$ after transfection for Igk mRNA and protein detection.

\section{Immunofluorescence}

Frozen sections of heart tissues, primary cultured cardiomyocytes, and HL-1 cells were fixed with acetone, and blocked with 5\% FBS at room temperature for 30 minutes. The cells were then stained overnight at $4{ }^{\circ} \mathrm{C}$ with the following primary antibodies: rabbit anti-mouse Igk (1:50; Proteintech), rabbit anti-mouse Cx43 (1:100; Proteintech), rabbit anti-mouse N-cadherin (1:100; Proteintech), rabbit antimouse Plakoglobin (1:100, Proteintech) were added at $4^{\circ} \mathrm{C}$ overnight. Then, the cells were washed with PBS and incubated for $1 \mathrm{~h}$ with the respective secondary antibodies conjugated to 5 (1:1000; Abcam), Alexa Fluor 568 (1:1000; Abcam) or Alexa Fluor 594 (1:1000; Abcam) after which the cells were stained with Hoechst (1:10000; Sigma) for $5 \mathrm{~min}$ at room temperature.

\section{Western blot analysis}

Total cellular protein was extracted from heart tissue and HL-1 cells using RIPA lysis buffer (Beyotime) containing a protease inhibitor cocktail. The samples were subsequently separated by $12.5 \%$ SDS-PAGE and transferred onto nitrocellulose membranes. The membranes were then incubated with the following primary antibodies: Biotin-labeled goat anti-mouse Igk (1:1000; SouthernBiotech), rabbit anti-mouse Cx43 (1:1000; Proteintech), rabbit anti-mouse N-cadherin (1:1000; Proteintech), rabbit anti Desmoplakin (1:1000, Proteintech), GAPDH (1:1000, ZSGB-Bio) and b-actin (1:1000, ZSGB-Bio). The secondary antibodies conjugated with HRP (ZSGB-Bio) were applied at a concentration of 1:10,000 and visualized using with ECL chemiluminescence (GE). Western Blots were quantified by densitometry using ImageJ software.

\section{Generation of conditional cardiac-specific Igk-knockout mice}

To generate inducible cardiac-specific lgk cKO mice, transgenic mice expressing a tamoxifen-inducible Cre recombinase protein under the control of the a-myosin heavy chain promoter, aMHC/MerCreMer mice (MCM) were intercrossed with Igk flox/flox mice (obtained from Shanghai Biomodel Organism Science \& 
Technology Development Co., Ltd) in a C57BL/6 genetic background. To induce Cre recombination, 8- to 12-week-old male aMHC/MerCreMer; Igk ${ }^{\text {flox/flox }}\left(\mathrm{Cre}^{+} ; \mathrm{Igk}^{\mathrm{f} / \mathrm{f}}\right)$ were treated with $80 \mathrm{mg} / \mathrm{kg}$ of tamoxifen (Sigma) via intraperitoneal injection once a day for 4 consecutive days. aMHC-Cre; $\operatorname{lgk}^{\mathrm{f} / \mathrm{f}}$ mice, aMHC$\mathrm{Cre}^{+}$mice with tamoxifen age- and sex-matched littermates were utilized as control mice. Conditions of animal housing and all experimental procedures were conducted under institutional guidelines provided by the Institutional Animal Care and Use Committee of China.

\section{Construction of adenovirus}

The full-length cDNA of mouse lgkV17-121/J2/C was inserted into adeno-associated virus serotype 9 vector-cTnT (AAV9-cTnT) to construct the AAV9-cTnT-Igk virus (produced by Likeli, Beijing, China). $5 \times 10^{11} \mathrm{vg}$ of AAV9-cTnT-Igk or AAV9-CMV-GFP were injected intravenously into 10-week-old cardiac lgk

knockout mice (aMHC-Cre ${ }^{+} \mathrm{Igk}^{\mathrm{fl} / \mathrm{fl}}$ ), while the same volume of AAV9-CMV-GFP was injected intravenously into aMHC-Cre ${ }^{+}$mice. Four weeks after injection, tamoxifen was injected to induce lgk gene knock down. Western blot of myocardial tissues was performed to confirm the re-expression efficiency.

\section{Systolic blood pressure analysis}

Systolic blood pressure was measured in conscious mice using BP-98A Blood Pressure Analysis System (Softtron). Briefly, animals were placed in a plastic chamber maintained at $37^{\circ} \mathrm{C}$, and a cuff with a pneumatic pulse sensor was attached to the tail. we measured systolic blood pressure five times per animal and recorded the mean overall blood pressure.

\section{Echocardiography analysis.}

To evaluate left ventricular function and dimension, transthoracic two-dimensional echocardiography was performed on mice sedated with 1.5\% isoflurane using a Visual Sonics Vevo 770 ultrasound system (Visual Sonics) equipped with a 17.5-MHz linear array transducer. M-mode tracings in the parasternal short axis view were used to measure left ventricular posterior wall thicknesses (LVPW) and left ventricular inner dimension (LVID) at end-diastole, which were used to calculate left ventricular fractional shortening (FS) and the ejection fraction (EF) according to standard formulas.

\section{Sarcomere shortening analysis}

Isolated cardiomyocytes were placed on the experimental chamber of an inverted microscope (Olympus) with permanent perfusion of Tyrode solution containing $500 \mathrm{mM} \mathrm{Ca}^{2+}$ heated to $37^{\circ} \mathrm{C}$. Myocytes were electrically stimulated $(15 \mathrm{~V}, 0.5 \mathrm{hz})$ and sarcomere shortening was detected using a videobased detection system (IonOptix) on intact myocytes. Six twitches per myocyte were collected for each sample $(n=2)$. Signal averaged data were analyzed to determine resting sarcomere length, peak shortening normalized for resting sarcomere length (percent peak height), time to peak shortening (TTP).

\section{Electrocardiography analysis}


Surface electrocardiogram (ECG) recordings were performed on mice anaesthetized with $1-1.5 \%$ pentobarbital sodium. Needle electrodes were used and placed in the conventional lead II position. A differential amplifier amplified the signals in the bandwidth of $0.1-1000 \mathrm{~Hz}$, and signals were filtered using an adaptive $60 \mathrm{~Hz}$ filter. The signals were digitized at $3000 \mathrm{~Hz}$, and analyzed using ECG Analysis Software (QRS phenotyping, Calgary, Canada).

\section{Electron microscope analysis}

The heart tissues fixed with $3 \%$ glutaraldehyde were chopped into $1 \mathrm{~mm}^{2} \times 1 \mathrm{~mm}$ pieces. Then, the tissues were rinsed, dehydrated, embedded, sliced, stained, and subsequently investigated under a transmission electron microscope (TEM).

\section{Statistical analysis}

All data are presented as the means \pm s.e.m. Statistical analyses were performed using Prism Software. The statistical significance of the difference between two sets of data was assessed using an unpaired ttest. Statistical significance was defined as $P<0.05$. ${ }^{\star} P<0.05$, ${ }^{\star *} P<0.01, * \star * P<0.001$.

\section{Data Availability}

This study includes no data deposited in external repositories

\section{Results}

\section{Igk expression in mouse and human cardiomyocytes}

To determine whether Igk is present in cardiomyocytes in the mice, immunofluorescence and immunogold electron microscopy were performed by using anti-mouse Igk antibodies. Positive staining for Igk was clearly observed in cardiomyocytes, especially on intercalated discs (ICDs) in heart tissue (Fig $1 \mathrm{Aa}, \mathrm{C}$ ), and Igk was colocalized with plakoglobin, which is a specific marker of ICDs (Fig 1Ab). Given that $B$ cells are considered the only sources of Igs, B cell-deficient mice (mMT mice) were used to exclude disturbance of B-Ig. As expected, Igk was also observed in the cardiomyocytes of mMT mice by immunofluorescence and Western blotting. As shown in Fig 1Aa and Fig 1B, Igk was found on ICDs in mMT hearts, and the signal intensity was comparable with that in WT mice. This indicates that Igk on ICDs originated from non-B cells. Subsequently, HL-1 myocardial cells and primary cultured cardiomyocytes from newborn mice were used to further confirm the existence of Igk in cardiomyocytes, as illustrated in Fig 1C and Fig 1D. Igk was localized on the cross-striations of primary cultured cardiomyocytes from neonatal mice and the cell junctions of HL-1 cells. Moreover, knockdown of Igk by siRNA resulted in reductions in Igk levels and Igk staining at cell junctions, suggesting that Igk can be produced by the cardiomyocytes of mice (Fig 1D). To demonstrate that Igk can also be expressed in human cardiomyocytes, 3 normal hearts and 3 viral myocarditis hearts obtained from autopsies were used for immunohistochemical staining, and Igk was found to be localized on ICDs of cardiomyocytes. 
To further confirm that Igk is produced by human cardiomyocytes, Igk was detected in human iPS-CM (induced pluripotent stem cell-derived cardiomyocytes) by IF and RT-PCR; significant staining for Igk was observed on the cell membrane, and Igk was found to be colocalized with plakoglobin (Fig 1E).

Surprisingly, we identified free Igk ( $\mathrm{k}$ free light chain, $\mathrm{kFLC}$ ) as the main form of Igkin heart tissue, as revealed by nonreduced SDS-PAGE/Western blotting (Fig 1Fa). Interestingly, ischaemia promoted the secretion of $\mathrm{k}$ FLC in cardiomyocytes (Fig $1 \mathrm{Fb}, \mathrm{c}$ ).

\section{Igk transcripts in cardiomyocytes with restricted diversity compared with that of B cells}

To further identify whether Igk was synthesized by cardiomyocytes, the rearranged transcripts of Igk were detected in cardiomyocytes by RT-PCR using primers for the variable regions and constant regions of the Igk gene. First, adult cardiomyocytes were isolated from wild-type (WT) or mMT (B cell-deficient) mice, and the rearranged Igk transcripts were successfully amplified from cDNA libraries of cardiomyocytes (Fig 2Aa, b). Sequencing analysis showed that all the cardiomyocyte-derived Igk transcripts we analysed had classical V-J rearrangements. Interestingly, unlike the B cell-derived Ig that showed high diversity, the $V-J$ rearrangement pattern of cardiomyocytes showed very restricted diversity (Fig 2Ac, d). Similarly, the V-J rearrangement pattern in cultured cardiomyocytes isolated from neonatal mice showed a conservative sequence that was different from that in fibroblasts isolated from the same heart (supplementary Fig 1A, B). In addition, the Igk transcript was also detected in HL-1 cells, with IgkV17-121/J2 being the dominant VJ pattern (Fig 2C).

Subsequently, single cardiomyocyte RNA-seq analysis was applied to further confirm Igk expression and sequence characteristics. Single cardiomyocytes from either WT or MMT mice were manually picked under microscopy by a mouth pipette. Cardiomyocytes were easily distinguished by and picked based on their unique rod-like cell shapes, and total RNA was obtained and reverse transcribed into CDNA according to Tang's masterpiece (supplementary Fig 1C). Then, CD20 transcripts were amplified to exclude singlecell cDNA contamination from B cells, and Myh6, cTnT and cTnl transcripts were amplified to identify cardiomyocyte. Additionally, Igk transcripts were sequenced by Sanger sequencing and next-generation sequencing. We isolated 56 single cardiomyocytes from both WT and mMT mice and found that $73 \%$ $(42 / 56)$ of single cardiomyocytes showed typical rearranged lgk sequencing (Fig 2Da, b, supplementary Fig 1D, F). Interestingly, we found that $30 \%$ (13/42) of single cardiomyocytes expressed more than one functional VkJk pattern (Fig 2D f). We compared the frequency of $\mathrm{Vk}$ and $\mathrm{Jk}$ usage in cardiomyocytes with that of $B$ cells and found that VJ usage was quite different between cardiomyocytes and $B$ cells. The cardiomyocyte-derived Igk gene showed an obvious restricted pattern of VJ combinations. In detail, the dominant Vk usages in cardiomyocytes were IgkV17-121 (28.3\%, 95/336), IgkV9-120 (25\%, 84/336), and IgkV14-100 (15.5\%, 52/336), but low frequency was used by B cells (Vk17-121 (2.1\%), Vk9-120 (2.5\%) and Vk14-100 (0.6\%)) (data from https://www.10xgenomics.com/). However, the B cell-derived Vk pattern showed diversity, and the dominant Vk usage in B cells was Vk10-96 (6\%), which was not detected in cardiomyocytes (Fig 2Dc-h). More interestingly, Vk17-121 and Vk9-120 localize next to each other on the genome, suggesting that this chromosomal region may be open and more easily 
transcribedsupplementary Fig 1E). Additionally, we also analysed the sequence of the $\mathrm{J}$ segment usage and $\mathrm{V}-\mathrm{J}$ junction between $\mathrm{CM}$-lgk and $\mathrm{B}$-lgk and found that the dominant usage of the $\mathrm{J}$ segment is $\mathrm{Jk} 1$ in $\mathrm{B}$ cells and $\mathrm{Jk} 2$ in cardiomyocytes (Fig 2Di). The V-J junction analysis showed that the sequences of the V-J junction in IgkV9-120/J2 and IgkV14-100/J2 were quite the same between CM-Igk and B-Igk, Interestingly, the sequences of the $\mathrm{V}-\mathrm{J}$ junction in IgkV17-121/J2 were quite different between CM-Igk and B-Igk, with 63\% (46/73) of the CM-IgkV17-121 segment and J2 segment showing a direct connection; however, there was a deletion of nucleotides in the $\mathrm{V}$ terminal and $\mathrm{J}$ terminal at the $\mathrm{V}-\mathrm{J}$ junction of $\mathrm{B}$ IgkV17-121/J2 (66/91) (supplementary Fig 1G).

\section{Cardiomyocyte-specific Igk knockout results in decreased cardiac conduction and contractility}

To evaluate Igk function in cardiomyocytes, a tamoxifen-induced Igk cKO mouse was established by intercrossing $\mathrm{Igk}^{\mathrm{f} / \mathrm{f}}$ mice with a-MHC-Cre mice (Fig 3A). Igk cKO mice and their littermates (8- to 12-weekold), including $\mathrm{Igk}^{\mathrm{f} / \mathrm{f}}$ and a-MHC-Cre mice, were administered tamoxifen for four consecutive days. Two days after tamoxifen treatment, we first analysed cardiac function by analysing systolic blood pressure, and unexpectedly, we found that cKO mice displayed significantly reduced blood pressure (Fig 3C), indicating that heart function was impaired. Additionally, echocardiographic analysis showed that the contractility of the cKO mice was markedly impaired compared with that of the control mice, with cKO mice showing reductions in ejection fraction (\% EF; $51.87 \% \pm 4.355$ for cKO mice versus $68.26 \% \pm 2.219$ for control mice) and LV fractional shortening (\% FS; $24.91 \% \pm 3.061$ for cKO mice versus $36.52 \% \pm 1.584$ for control mice) (Fig 3D). Moreover, a contractile shortening assay showed that isolated single cardiomyocytes from cKO mice exhibited a reduced contractile response compared with those from control mice $(0.0422 \pm 0.006719$ for cKO mice versus $0.1520 \pm 0.04271$ for control mice) (Fig 3E). More importantly, we found that ablation of Igk in cardiomyocytes led to the death of $40 \%$ cKO mice as early as $\mathrm{d} 4(\mathrm{n}=16)$. In contrast, tamoxifen-treated control mice $\left(\operatorname{lgk}^{\mathrm{f} / f}\right)$ appeared healthy throughout the course of tamoxifen treatment (Fig 3B). These data suggest that the expression of Igk in cardiomyocytes is critical for heart function.

\section{Cardiac-specific Igk knockout causes structural abnormalities of intercalated discs}

Intercalated discs are made up of short processes extending from two adjacent branches of myocardial fibers, which can spread excitation from one cell to another and facilitate synchronous contraction of myocardial fibers. Subsequently, we evaluated the electrophysiological activities of cardiomyocytes by electrocardiogram (ECG) and found that cKO mice exhibited a significant reduction in resting heart rate and QRS interval prolongation (Fig 3F). To further examine the significance of the reduced Igk levels and cardiac dysfunction triggered by cardiac stress, cKO and control mice were subcutaneously injected with isoproterenol (ISO). As expected, ISO injection induced more frequent atrioventricular (AV) block in cKO mice $(6 / 6)$ than in control mice (1/6). Furthermore, unlike in control mice, ISO did not significantly increase the heart rate of cKO mice, which indicated that Igk deficiency decreases the response of cardiomyocytes to ISO. 
Given that Igk is localized on ICDs and Igk deficiency induced severe conduction dysfunction, we next used transmission electron microscopy (TEM) to analyze the ultrastructural of ICDs in the hearts of cKO and control mice. We found that the structures of the ICDs of CKO mice were highly convoluted and disorganized with widened gaps from day 2 after tamoxifen administration (Fig 4A). However, normal ICD structures with clear adherens junctions and desmosomes were visible in the hearts of control mice. The results indicated that Igk plays a very important role in maintaining ICD structure. The components of ICDs, namely, plakoglobin (a cytoplasmic desmosomal protein), N-cadherin (an adherens junction protein) and connexin 43 (a gap junction protein), were examined by immunofluorescence staining and stimulated emission depletion microscopy (STED). Our results showed that depletion of Igk in ICDs resulted in a significant decrease in N-cadherin and $\mathrm{Cx} 43$ staining in ICDs, while plakoglobin staining was increased in the ICDs of cKO mice compared to control mice (Fig 4B, C, D, E, supplementary Fig 2). Interestingly, an obvious decrease in N-cadherin occurred on the third day after tamoxifen administration, while $\mathrm{Cx} 43$ was decreased on the ICD on the fourth day. Moreover, Western blot analysis further confirmed a reduction in $\mathrm{Cx} 43$ and $\mathrm{N}$-cadherin in the hearts of cKO mice compared with control mice (Fig $4 \mathrm{~F})$. These results indicated that Igk is required to maintain the localization of $\mathrm{N}$-cadherin and $\mathrm{Cx} 43$ on ICDs.

To further confirm Igk function in ICDs, we overexpressed Igk 4 weeks before tamoxifen administration by generating AAV9-cTnT-Igk in Igk ${ }^{\mathrm{f} / \mathrm{f}}$;a-MHC-Cre ${ }^{+}$mice. Igk was knocked out in the cardiomyocytes of Igk $^{\mathrm{f} / \mathrm{f} ; \mathrm{a}-}$ $\mathrm{MHC}-\mathrm{Cre}^{+}$mice with tamoxifen. Rescue of Igk by AAV9-cTnT-Igk significantly improved cardiac function by increasing the \% EF (\% EF; $44.04 \pm 9.591$ for CKO mice versus $70.57 \pm 4.090$ for cKO mice injected with AAV9-cTnT-lgk and $73.34 \pm 4.473$ for control mice) and \% FS (\% FS; $21.89 \pm 5.217$ for cKO mice versus $40.01 \pm 3.379$ for cKO mice injected with AAV9-cTnT-Igk and $42.81 \pm 3.645$ for control mice) (Fig 5A) and reversed the destruction of ICD structure by upregulating the protein levels of Igk, DSP, N-cadherin and Cx43 (Fig 5B, C, supplementary Fig 3), confirming that dysfunction of cardiomyocytes and abnormal ICD structure of was caused by Igk deficiency.

\section{Cardiac-specific lgk knockout induces degradation of plectin, which causes disconnection of desmin and desmosomes}

To understand the mechanism of how Igk affects the structure and function of ICDs, we wanted to identify the proteins that interact with Igk in cardiomyocytes. Total heart tissue lysates were extracted, GST-Igk or GST (as a control) was used to identify the proteins that interact with Igk by GST pull down, and protein mass spectrometry analysis was used to identify the potential interacting proteins. Unlike in the control group, desmoplakin and its binding protein plakoglobin bound with GST-Igk in the ICDs of Igk cKO mice (Fig 6A), suggesting that Igk interacts with desmoplakin. Desmoplakin associates with plakoglobin, plakophilin, b-catenin and a-catenin to form a complex that connects desmosomes and adherens junction (AJs) within the 'area composite' and work together to connect ICDs to the cytoskeleton, especially actin and intermediate filaments. Disconnection of actin or desmin with ICDs leads to a loss of desmosomes and AJ components ${ }^{28,29}$. Therefore, we also determined the localization of desmin and actin in cardiomyocytes, and the immunofluorescence results showed that staining for 
desmin and actin on ICDs was diminished in heart tissues from Igk cKO mice compared to control mice (Fig 6Ba, c). Similarly, the filamentous structures of desmin and F-actin in HL-1 cells was abolished when Igk was knocked down by specific siRNA (Fig 6Bb, d), suggesting that a lack of Igk led to the lost connection between two cytoskeleton components actin and desmin and ICDs. In our previous finding, we found that cancer cell-derived Igk interacts with the cytolinker protein plectin and that a lack of Igk leads to the disappearance of plectin and disassembly of actin as well as intermediate filaments. Plectin is a 500-kDa cytoskeleton-associated protein, and the C-terminus of Plectin can link the intermediate filaments to the transmembrane proteins of hemidesmosomes and maintain cell-to-cell connectivity. Moreover, the N-terminus of plectin can link actin to promote its assembly, and a lack of plectin can lead to disassembly of intermediate filaments and actin ${ }^{30,31}$. Moreover, it has been reported that plectin can connect desmin with desmoplakin on $\mathrm{ICDs}^{32,33}$. Thus, we analysed whether there is an interaction between Igk and plectin on ICDs. The immunofluorescence results revealed that Igk and plectin were colocalized on the ICDs of cardiomyocytes, as well as on the cell junctions of HL-1 cells (Fig 6Da). Knockdown of Igk led to decreased plectin and loss of localization on ICDs (Fig 6Db-d). Next, we established an expression vector containing the C-terminus of plectin, and IP assays showed that Igk interacts with both desmoplakin and plectin to form a complex (Fig $6 \mathrm{C}$ ). The results of our protein stability experiments using cyclohexane $(\mathrm{CHX})$ to inhibit protein synthesis revealed that lgk can maintain the stability of plectin, which can explain how Igk knockdown results in the lost connection between cytoskeleton and ICDs (Fig 6E).

\section{Discussion}

We reported for the first time that the Igk light chain, which is currently believed to be a classical immune molecule, can be widely expressed in both mouse and human cardiomyocytes. Functionally, cardiomyocyte-derived k-type light chain, as a novel structural protein of cardiomyocytes, mainly plays an important role in maintaining the normal structure of cardiomyocyte intercalated discs and normal myocardial contraction and conduction.

In fact, there are many clues that cardiomyocytes can produce Igs, but due to the limitations of classical immunological theory, researchers still believe that Igs are produced by B cells. However, there are many contradictory phenomena that cannot be well explained by classical immunological theory. For example, in acute myocardial infarction, increased free Ig light chain or IgE appears rapidly in the blood, which is not consistent with the law of antibody production of B cells; some myocardial injury diseases are associated with high levels of "autoantibodies against cardiac structural proteins", but to date, scientists have not found a B cell clone that can produce these autoantibodies. Our findings suggest that the "autoantibodies against cardiac structural proteins" in myocardial injury diseases may be produced by injured cardiomyocytes and may be involved in unidentified activities rather than acting as autoantibodies. Our previous work revealed that cardiomyocytes can widely express different classes of Ig, including $\operatorname{lgM}{ }^{24}$, IgG and IgE, as well as the Igk light chain, but their physiological function remains unclear. Therefore, in this study, we focused on Igk to reveal its physiological function in cardiomyocytes. 
In this study, we first showed evidence of Igk expression in cardiomyocytes at the protein and transcription levels. Our results revealed that Igk was widely expressed in mouse and human cardiomyocytes; moreover, Igk was mainly located on the intercalated disc. Subsequently, we analysed the characteristics of variable regions of cardiomyocyte-derived lgk. It is well known that the lg variable region produced by $B$ cells is infinitely diverse because its main function is to bind to a variety of antigen molecules, such as antibodies or BCR. Therefore, we first analysed whether the characteristics of the cardiomyocyte-derived Igk variable region are different from those of Igk in B cells. Our single cell sequencing results showed that, unlike B cell-derived Igk, which shows diversity, cardiomyocyte-derived Igk exhibited a very conservative VkJk usage and pattern and tended to express IgkV17-121 (28.3\%, 95/336), IgkV9-120 (25\%, 84/336), and IgkV14-100 (15.5\%, 52/336) in cardiomyocytes in adult mice. It is worth noting that the conservative characteristics of cardiomyocyte-derived Igk were similar to those of Igk in hepatocytes and other non-B cells, especially IgkV9-120, which has been found to be highly frequently expressed in other non-B cell lineages ${ }^{34,35}$. However, the expression frequency of the above VkJk usages in B cells was less than $3 \%$. This limited diversity suggests that cardiomyocyte-derived $k$ light chain may not participate as an antibody in humoural immunity but may play another important physiological role.

Next, we constructed conditional mice with conditional Igk knockout in cardiomyocytes (lgk f/f; aMHC$\mathrm{Cre}^{+}$mice) to determine the physiological function of Igk. To prevent the occurrence of I light chain redundancy in the early development of mouse cardiomyocytes, the conditional Igk knockout was inducible, allowing the knockout of the lgk gene from cardiomyocytes at any stage of mouse development by tamoxifen treatment. In this study, we selected 8-week-old mice for conditional knockout. We first found that compared to control $\left(\mathrm{Igk}^{\mathrm{f} / \mathrm{f}}\right.$ or aMHC-Cre $\left.{ }^{+}\right)$mice, Igk knockout mice exhibited significant decreases in blood pressure, cardiac ejection ability, and heart rate from day 2 after the administration of tamoxifen. Isoproterenol (ISO) was used to increase cardiac oxygen consumption and heart rate and led to atrioventricular block. Importantly, Igk knockout resulted in the death of over $40 \%$ of Igk cKO mice from day 4 after the administration of tamoxifen, suggesting that lgk is involved in maintaining normal cardiac contraction and conduction function.

To evaluate the mechanism, we observed changes in myocardial structure in Igk cKO mice. Our results showed that Igk knockout led to disordered intercalated disc structure and loss of localization of $\mathrm{N}$ cadherin, an adhesion junction effector, and $\mathrm{Cx} 43$, a gap junction effector, at intercalated discs, suggesting that the abnormal myocardial contraction and conduction caused by Igk knockout may have been caused by structural disorder of the intercalated discs. To further confirm this result, we used AAV9cTnT-Igk to overexpress Igk. The results showed that when cardiomyocytes overexpressed Igk, knockout induced by tamoxifen did not cause disorder of cardiomyocyte structure or function, suggesting that the above phenomenon was indeed caused by lgk knockout in cardiomyocytes.

ICDs are composed of three major complexes: desmosomes, adherens junctions (AJs) and gap junctions (GJ)s. Desmosomes and AJs provide mechanical attachment between two cardiomyocytes by anchoring the actin cytoskeleton and intermediate filaments (IFs), enhancing the stability of the ICD. GJs connect 
the cytoplasm of adjacent cardiomyocytes metabolically and electronically to enable the propagation of electrical stimuli throughout heart muscle cells. These three complexes work together to incorporate the mechanical and electrical functions of the ICD ${ }^{36-39}$. Notably, components of desmosomal and adherens junctions are intimately associated within the area composita and work together to facilitate mechanical coupling. In the area composita, desmosomes are considered to be associated with the intermediate filament desmin through binding desmoplakin, and AJs are considered to be associated with actin through binding a-catenin, b-catenin and vinculin ${ }^{28,29}$. However, how does Igk knockout affect the localization and expression of ICD-related proteins? Our study found that Igk can interact with desmoplakin. Igk knockout first led to a reduction in N-cadherin and then in Cx43 and a loss of localization on ICDs, suggesting that Igk knockout first affects desmosome and AJ function and then secondarily affects GJs. To address why lgk knockout results in instability of ICDs, we further determined the intracellular localization of both F-actin and desmin and found that Igk knockout also resulted in disassembly of both F-actin and desmin. The results suggest that disassembly of both F-actin and desmin may be the cause of instability of ICDs resulting from Igk knockout. To date, no evidence has indicated that desmoplakin function is related to actin, which suggests that there may be other proteins related to Igk; this study suggested that desmoplakin interacts with Igk. Plectin is a large cytolinker protein, and its main function is to interact and promote the assembly of actin and intermediate filaments 40-43. It has been reported that plectin is localized on ICDs and connects intermediate filaments/desmin to desmosomes by interacting with desmoplakin ${ }^{32,33}$. Moreover, our previous work demonstrated that cancer cell-expressed Igk can interact with plectin (unpublished). Based on the above evidence, we hypothesize that cardiomyocyte-expressed Igk can interact with plectin, which may be involved in the stability of ICDs. Our results showed that Igk can interact with plectin and that Igk gene knockout results in a significant loss of plectin and disassembly of desmin and F-actin. Moreover, a loss of plectin leads to disruption of AJs and GJs, which leads to abnormal electrical coupling of cardiomyocytes and reduced conduction velocity. Our mechanistic study revealed that Igk can promote the stability of plectin on ICDs. However, the mechanism by which Igk regulates plectin and its role in ICD assembly still require further investigation.

Our study has only broken the ice, and it is believed that there are many physiological and pathological functions of cardiomyocyte-expressed Igs that have yet to be revealed. For example, myocardial amyloidosis is a common heart disease that is caused by pathogenic FLCs. At present, it is generally accepted that these pathogenic Ig light chains are secreted and deposited into myocardial tissue by multiple myeloma cells; however, in most cases, no malignant proliferation of plasma cells is found in myocardial amyloidosis. In contrast, we found that cardiomyocytes can secrete FLC under conditions of cell stress, such as hypoxia. Our study provides novel insight into the source of abnormally elevated FLC in myocardial amyloidosis and myocardial injury, such as myocardial infarction and viral myocarditis ${ }^{44}$. However, the exact pathological mechanism of cardiomyocyte-derived lgs is still unclear and requires further investigation.

\section{Declarations}


Acknowledgments

We thank Prof. Zhihai Qin (Institute of Biophysics, Chinese Academy of Sciences) for providing us with the $\mu \mathrm{MT}$ mice and Prof. W.C. Claycomb (Louisiana State University, New Orleans, LA) for providing us with HL-1 cell line. We are grateful to Ming Zheng (Department of Physiology and Pathophysiology, Health Science Center, Peking University) and Yao Song (Institute of Vascular Medicine, Peking University Third Hospital) for technical assistance and helpful discussion.

\section{Sources of Funding}

This work was supported by research grants to X. Qiu from the key support projects of the National Natural Science Foundation's major research program

(91642206).

\section{Author contributions}

ZZ and QXY conceived the study and designed the workplan. Experimental work: ZZ, SZ, ZWJ. Development and contribution of reagents: LF, LQY. Manuscript writing: ZZ and QXY.

\section{Conflict of interest}

None

\section{References}

1. Li, M. et al. Expression of immunoglobulin kappa light chain constant region in abnormal human cervical epithelial cells. Int J Biochem Cell Biol. 36, 2250-2257 (2004).

2. Babbage, G., Ottensmeier, C. H., Blaydes, J., Stevenson, F. K. \& Sahota, S. S. Immunoglobulin heavy chain locus events and expression of activation-induced cytidine deaminase in epithelial breast cancer cell lines. Cancer Res. 66, 3996-4000 (2006).

3. Chen, Z. \& Gu, J. Immunoglobulin G expression in carcinomas and cancer cell lines. The FASEB Journal. 21, 2931-2938 (2007).

4. Zheng, $\mathrm{H}$. et al. Expression and secretion of immunoglobulin alpha heavy chain with diverse VDJ recombinations by human epithelial cancer cells. Mol. Immunol. 44, 2221-2227 (2007).

5. Zhu, X. et al. Immunoglobulin mRNA and protein expression in human oral epithelial tumor cells. Appl Immunohistochem Mol Morphol. 16, 232-238 (2008).

6. Huang, J. et al. Expression of immunoglobulin gene with classical V-(D)-J rearrangement in mouse brain neurons. Int J Biochem Cell Biol. 40, 1604-1615 (2008).

7. Huang, J., Zhang, L., Ma, T., Zhang, P. \& Qiu, X. Expression of immunoglobulin gene with classical V(D)-J rearrangement in mouse testis and epididymis. J. Histochem. Cytochem. 57, 339-349 (2009). 
8. Shao, W. et al. Identification of Liver Epithelial Cell-derived Ig Expression in mu chain-deficient mice. Sci Rep. 6, 23669 (2016).

9. Jing, Z. et al. Expression of immunoglobulin $\mathrm{G}$ in human podocytes, and its role in cell viability and adhesion. Int. J. Mol. Med. 41, 3296-3306 (2018).

10. Deng, $\mathrm{H}$. et al. Expression of immunoglobulin $A$ in human mesangial cells and its effects on cell apoptosis and adhesion. Mol. Med. Rep. 17, 5272-5282 (2018).

11. Qiu, X. et al. Human epithelial cancers secrete immunoglobulin g with unidentified specificity to promote growth and survival of tumor cells. Cancer Res. 63, 6488-6495 (2003).

12. Sheng, Z. et al. Involvement of cancer-derived IgG in the proliferation, migration and invasion of bladder cancer cells. Oncol. Lett. 12, 5113-5121 (2016).

13. Tang, J. et al. Lung squamous cell carcinoma cells express non-canonically glycosylated IgG that activates integrin-FAK signaling. Cancer Lett. 430, 148-159 (2018).

14. Erdogan, O., Gul, C., Altun, A. \& Ozbay, G. Increased immunoglobulin E response in acute coronary syndromes. Angiology. 54, 73-79 (2003).

15. Shantsila, E., Tapp, L. D. \& Lip, G. Y. Free Light Chains in patients with acute coronary syndromes: Relationships to inflammation and renal function. Int. J. Cardiol. 185, 322-327 (2015).

16. Krijnen, P. A. et al. IgM colocalises with complement and $C$ reactive protein in infarcted human myocardium. J. Clin. Pathol. 58, 382-388 (2005).

17. Zhang, M. et al. The role of natural IgM in myocardial ischemia-reperfusion injury. J. Mol. Cell. Cardiol. 41, 62-67 (2006).

18. Jackson, C. E. et al. Combined Free Light Chains Are Novel Predictors of Prognosis in Heart Failure. JACC Heart Fail. 3, 618-625 (2015).

19. Dubrey, S. W. et al. The clinical features of immunoglobulin light-chain (AL) amyloidosis with heart involvement. QJM. 91, 141-157 (1998).

20. Liao, R. et al. Infusion of light chains from patients with cardiac amyloidosis causes diastolic dysfunction in isolated mouse hearts. Circulation. 104, 1594-1597 (2001).

21. Liao, R. et al. Infusion of light chains from patients with cardiac amyloidosis causes diastolic dysfunction in isolated mouse hearts. Circulation. 104, 1594-1597 (2001).

22. Levinson, R. T. et al. Role of mutations in the cellular internalization of amyloidogenic light chains into cardiomyocytes. Sci Rep. 3, 1278 (2013).

23. Kang, B. Y. et al. LOX-1 dependent overexpression of immunoglobulins in cardiomyocytes in response to angiotensin II. Biochem Biophys Res Commun. 379, 395-399 (2009).

24. Zhu, Z. et al. Immunoglobulin M, a novel molecule of myocardial cells of mice. Int J Biochem Cell Biol. 88, 172-180 (2017).

25. Claycomb, W. C. et al. HL-1 cells: a cardiac muscle cell line that contracts and retains phenotypic characteristics of the adult cardiomyocyte. Proc Natl Acad Sci U S A. 95, 2979-2984 (1998). 
26. Tang, F. et al. RNA-Seq analysis to capture the transcriptome landscape of a single cell. Nat. Protoc. 5, 516-535 (2010).

27. Shi, Z. et al. More than one antibody of individual B cells revealed by single-cell immune profiling. Cell Discov. 5, 64 (2019).

28. Manring, H. R., Dorn, L. E., Ex-Willey, A., Accornero, F. \& Ackermann, M. A. At the heart of inter- and intracellular signaling: the intercalated disc. Biophys Rev. 10, 961-971 (2018).

29. Zhao, G., Qiu, Y., Zhang, H. M. \& Yang, D. Intercalated discs: cellular adhesion and signaling in heart health and diseases. Heart Fail. Rev. 24, 115-132 (2019).

30. Castanon, M. J., Walko, G., Winter, L. \& Wiche, G. Plectin-intermediate filament partnership in skin, skeletal muscle, and peripheral nerve. Histochem. Cell Biol. 140, 33-53 (2013).

31. Staszewska, I., Fischer, I. \& Wiche, G. Plectin isoform 1-dependent nuclear docking of desmin networks affects myonuclear architecture and expression of mechanotransducers. Hum. Mol. Genet. 24, 7373-7389 (2015).

32. Pieperhoff, S. et al. The plaque protein myozap identified as a novel major component of adhering junctions in endothelia of the blood and the lymph vascular systems. J. Cell. Mol. Med. 16, 17091719 (2012).

33. Hoorntje, E. T. et al. No major role for rare plectin variants in arrhythmogenic right ventricular cardiomyopathy. Plos One. 13, e203078 (2018).

34. Shao, W. et al. Identification of Liver Epithelial Cell-derived Ig Expression in mu chain-deficient mice. Sci Rep. 6, 23669 (2016).

35. Shao, W. et al. Epithelial cells are a source of natural IgM that contribute to innate immune responses. Int J Biochem Cell Biol. 73, 19-29 (2016).

36. Gard, J. J. et al. Remodeling of gap junctions and slow conduction in a mouse model of desminrelated cardiomyopathy. Cardiovasc. Res. 67, 539-547 (2005).

37. Li, J. et al. Cardiac-specific loss of $\mathrm{N}$-cadherin leads to alteration in connexins with conduction slowing and arrhythmogenesis. Circ. Res. 97, 474-481 (2005).

38. Yang, Z. et al. Desmosomal dysfunction due to mutations in desmoplakin causes arrhythmogenic right ventricular dysplasia/cardiomyopathy. Circ. Res. 99, 646-655 (2006).

39. Li, J. et al. N-cadherin haploinsufficiency affects cardiac gap junctions and arrhythmic susceptibility. J. Mol. Cell. Cardiol. 44, 597-606 (2008).

40. Favre, B. et al. Plectin interacts with the rod domain of type III intermediate filament proteins desmin and vimentin. Eur. J. Cell Biol. 90, 390-400 (2011).

41. Eisenberg, J. L. et al. Plectin-containing, centrally localized focal adhesions exert traction forces in primary lung epithelial cells. J. Cell Sci. 126, 3746-3755 (2013).

42. Wiche, G., Osmanagic-Myers, S. \& Castanon, M. J. Networking and anchoring through plectin: a key to IF functionality and mechanotransduction. Curr. Opin. Cell Biol. 32, 21-29 (2015). 
43. Matsubara, T. et al. Regulation of osteoclast differentiation and actin ring formation by the cytolinker protein plectin. Biochem Biophys Res Commun. 489, 472-476 (2017).

44. Matsumori, A. et al. Effects of free immunoglobulin light chains on viral myocarditis. Circ. Res. 106, 1533-1540 (2010).

\section{Supplementary Materials}

\section{Supplementary Fig 1. Igk transcript was found in cardiomyocyte}

(A) a. Isolated cardiomyocyte of neonatal mice. Myosin was used to identify cardiomyocyte. Bar=20mm.

b. Transcript of Igk variable region and constant region in cardiomyocyte and fibroblast by RT-PCR. Bar $=20 \mathrm{~mm}$.

(B) Igk knockdown on mRNA levels in HL-1 cells by two independent siRNA and Igk transcript was detected by RT-PCR.

(C) a. Schematic overview of the single-cell sequencing workflow in cardiomyocyte. b. single cardiomyocyte was picked by a mouth pipette under microscopy.

(D) a. Transcript of Igk was detected in single cardiomyocyte. Spleen of WT mice was used as positive control. b. Transcript of CD20 was detected in single cardiomyocyte. Spleen of WT mice was used as positive control. c. Transcript of cTnT was detected in single cardiomyocyte. Heart tissue of WT mice was used as positive control.

(E) Igk V segment distribution on the chromosome.

(F) Sequence of Igk $\mathrm{J}$ region to $\mathrm{C}$ region which was amplified in sFig $1 \mathrm{C}$.

(G) Sequence of Igk CDR3 region of CM-Igk and B-Igk.

Supplementary Fig 2. Colocalization of N-cadherin/Cx43 and Plakoglobin on the ICD of Igk CKO and control mice.

(A) a. Colocalization of N-cadherin and plakoglobin on the ICD of cKO and control mice for consecutive four days after tamoxifen injection by confocal. B. Colocalization of Cx43 and plakoglobin on the ICD of CKO and control mice for consecutive four days after tamoxifen injection by confocal. Bar=25 mm.

\section{Supplementary Fig 3. Colocalization of N-cadherin/Cx43 and Plakoglobin on the ICD of cKO mice, control mice and CKO mice injected with AAV9-cTnT-Ig Igk vector.}

(A) a. Colocalization of $\mathrm{N}$-cadherin and plakoglobin on the ICD of cKO mice, control mice and cKO mice injected with AAV9-cTnT-Ig Igk. b. Colocalization of N-cadherin and plakoglobin on the ICD of cKO mice, control mice and cKO mice injected with AAV9-cTnT-Ig Igk. 
A
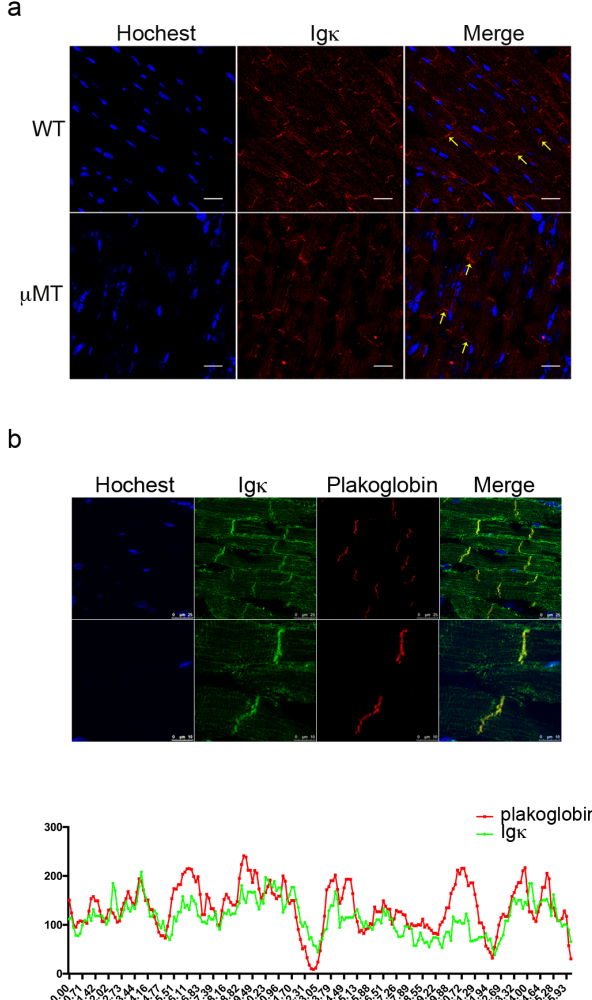

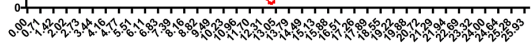
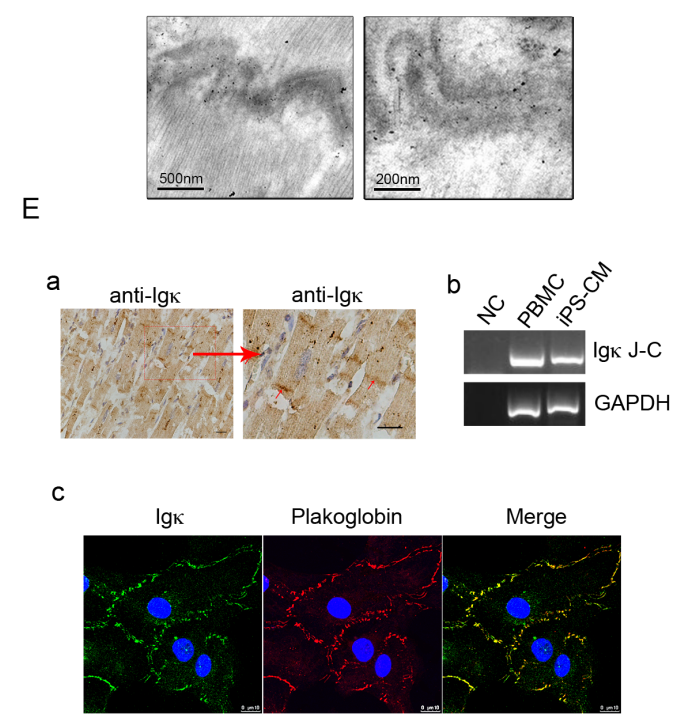

B

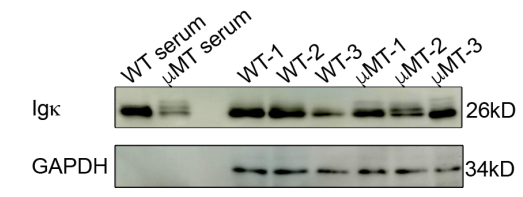

C

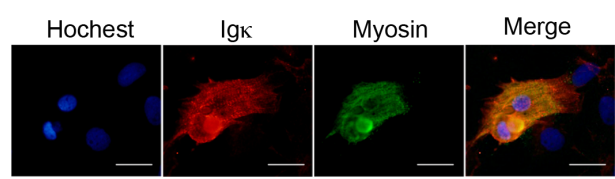

$\mathrm{D}_{\mathrm{a}}$

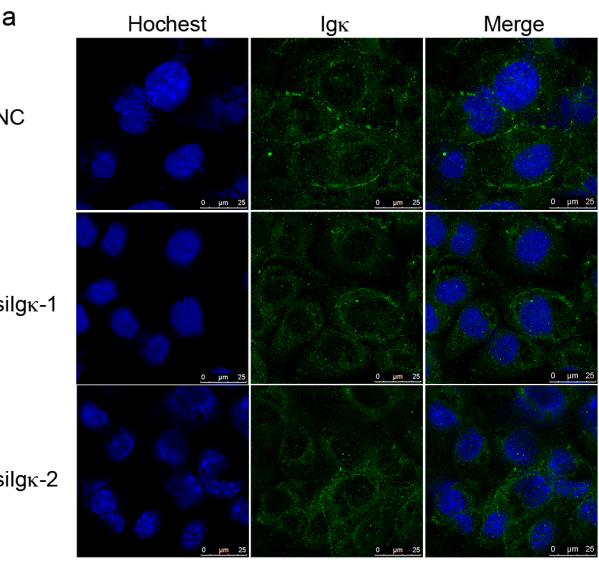

b

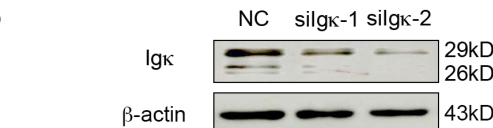

F

a

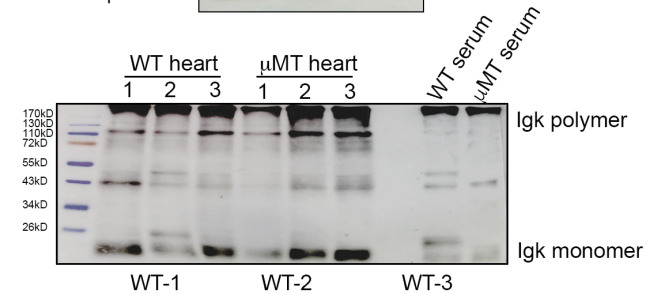

$b$
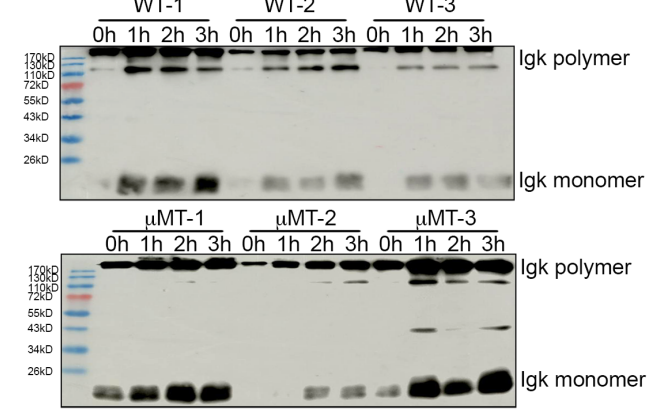

\section{Figure 1}

Presence of Igk molecules in cardiomyocyte of mice and human on the protein level (A) a. Igk staining (red) was detected in $\mu \mathrm{MT}$ and WT heart tissue by IF. Nuclei was detected with Hoechst (blue). Positive signal was found on the ICD of cardiomyocyte of both WT and $\mu \mathrm{MT}$ mice. Bar=75 $\mu \mathrm{m}$. b. Igk colocalized 
with plakoglobin on the ICD. Pearson's Correlation Rate $=0.5811$, Bar $=25 \mu m$. c. Igk positive signal was found on the ICD of cardiomyocyte of WT mice by immuno-electron microscope. (B) a. Igk expression was detected in heart tissue and serum of both $\mu \mathrm{MT}$ and WT mice by Western blot analysis under reduced condition, GAPDH was used as an internal control. (C) Igk staining (red) was detected on the cross striation of primary cultured cardiomyocyte of neonatal mice. Myosin(green) was used to identify

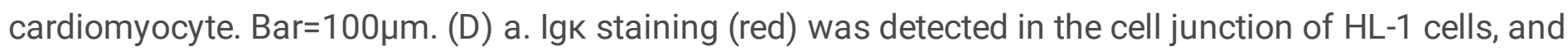
the positive signal was diminished by using two specific siRNA for lgk Bar=25 $\mu \mathrm{m}$. b. Igk knockdown on protein levels in $\mathrm{HL}-1$ cells by two independent siRNA. $\beta$-actin was used as an internal control. (E) a. Igk staining was detected on the ICD of human heart autopsy specimen by IHC. Bar=20 $\mu \mathrm{m}$. b. Igk staining was found to be colocalized with plakoglobin-a ICD marker of human iPS-CM. Bar=10 $\mu$ m. (F) a. b. Both

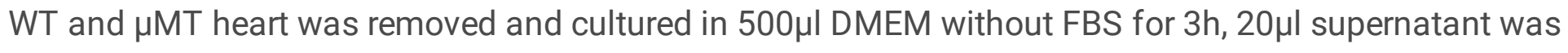
collected and detected for Igk under non-reduced condition per hour. 
A

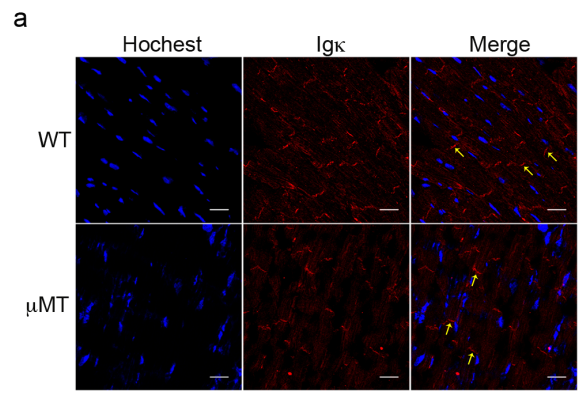

b
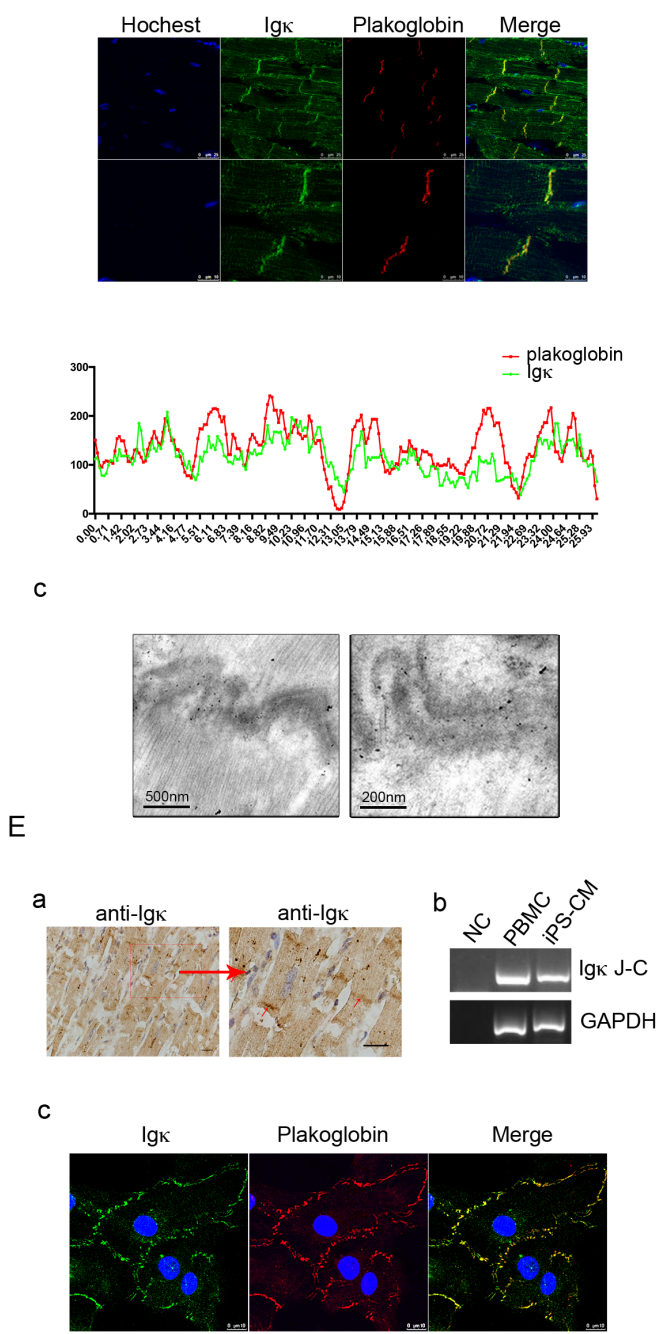

B

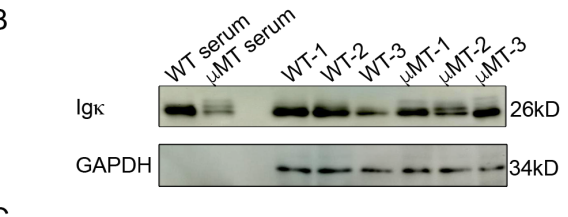

C

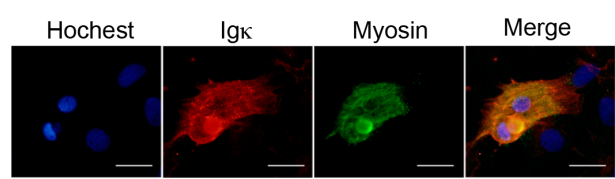

$\mathrm{D}_{\mathrm{a}}$

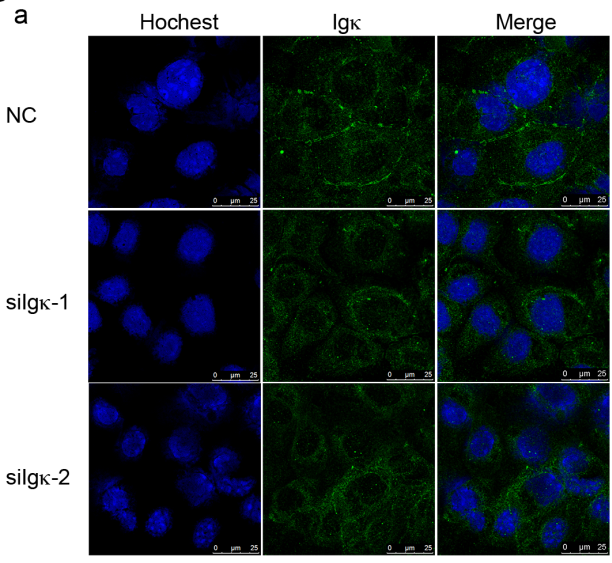

b

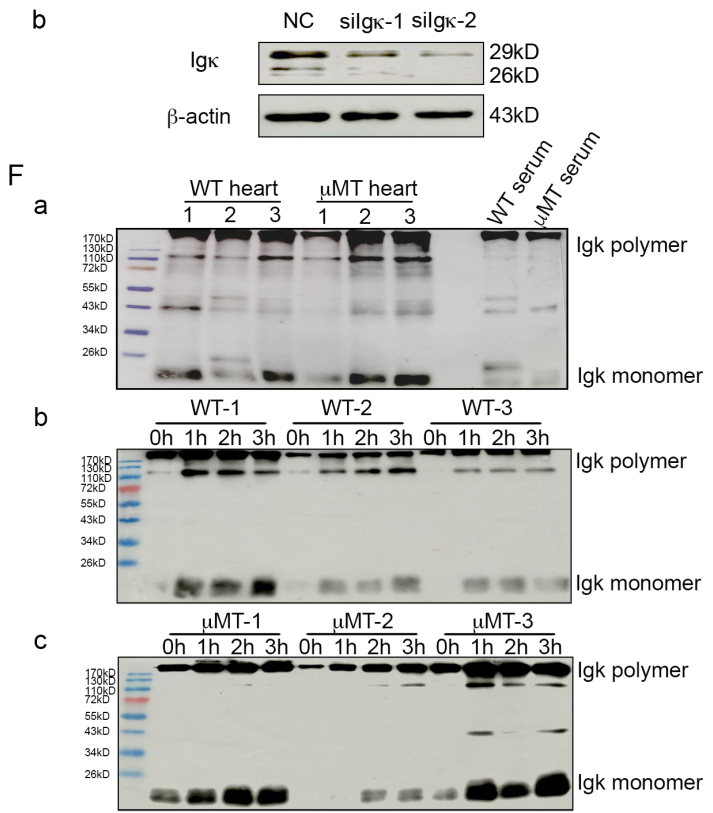

\section{Figure 1}

Presence of Igk molecules in cardiomyocyte of mice and human on the protein level (A) a. Igk staining (red) was detected in $\mu \mathrm{MT}$ and WT heart tissue by IF. Nuclei was detected with Hoechst (blue). Positive signal was found on the ICD of cardiomyocyte of both WT and $\mu \mathrm{MT}$ mice. Bar=75 $\mu \mathrm{m}$. b. Igk colocalized with plakoglobin on the ICD. Pearson's Correlation Rate $=0.5811$, Bar $=25 \mu \mathrm{m}$. c. Igk positive signal was found on the ICD of cardiomyocyte of WT mice by immuno-electron microscope. (B) a. IgK expression 
was detected in heart tissue and serum of both $\mu \mathrm{MT}$ and WT mice by Western blot analysis under reduced condition, GAPDH was used as an internal control. (C) Igk staining (red) was detected on the cross striation of primary cultured cardiomyocyte of neonatal mice. Myosin(green) was used to identify

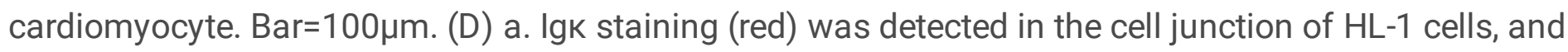
the positive signal was diminished by using two specific siRNA for lgk Bar $=25 \mu \mathrm{m}$. b. Igk knockdown on protein levels in HL-1 cells by two independent siRNA. $\beta$-actin was used as an internal control. (E) a. Igk staining was detected on the ICD of human heart autopsy specimen by IHC. Bar=20 $\mu \mathrm{m}$. b. Igk staining

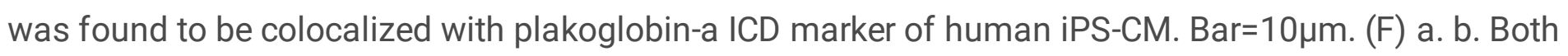
WT and $\mu \mathrm{MT}$ heart was removed and cultured in $500 \mu \mathrm{l}$ DMEM without FBS for $3 \mathrm{~h}, 20 \mu \mathrm{l}$ supernatant was collected and detected for Igk under non-reduced condition per hour. 


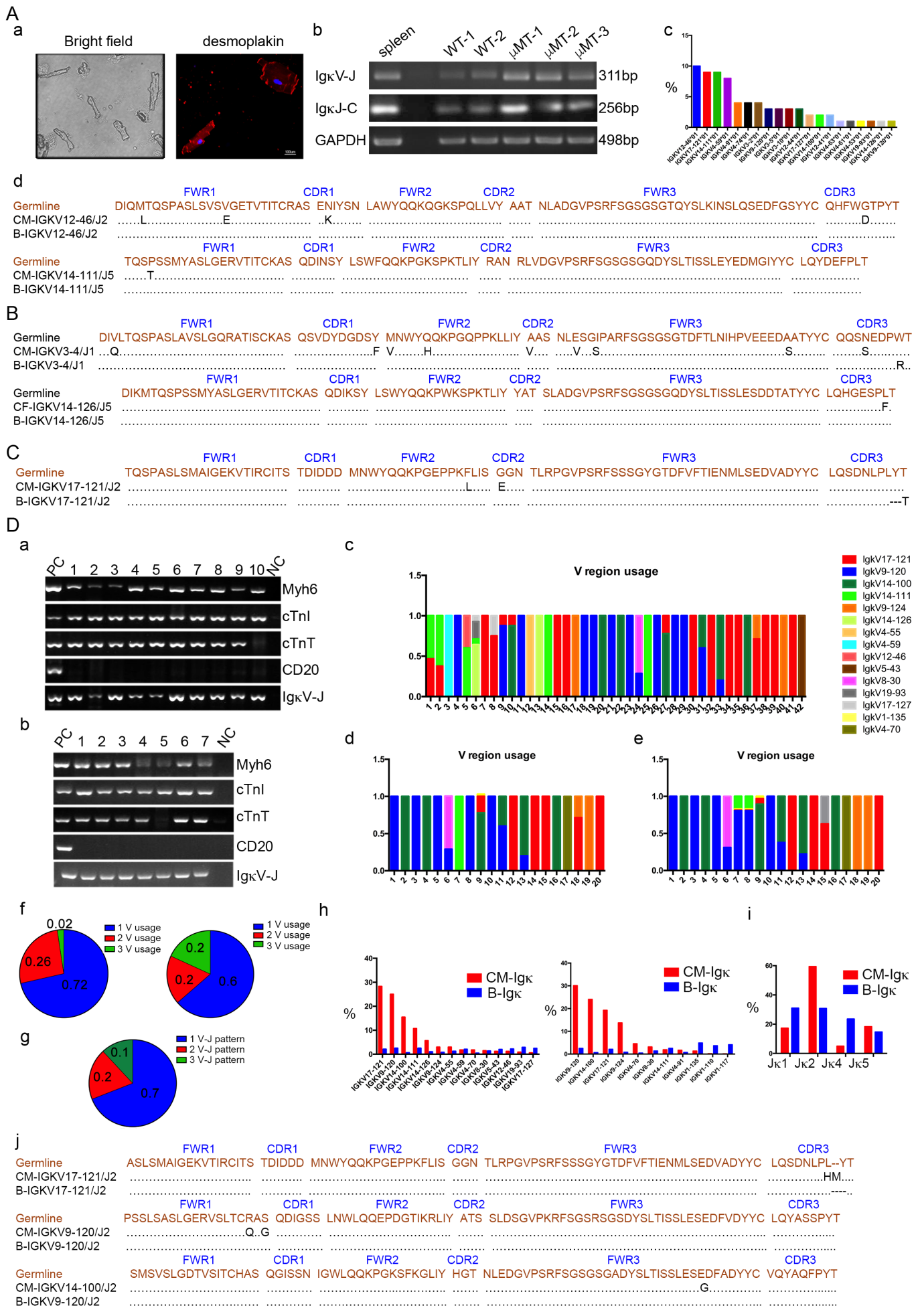

Figure 2

Igk transcripts were expressed in cardiomyocyte with restricted diversity compared with B cell (A) a. Isolated cardiomyocyte of adult mice under the bright field of microscopy(left). Desmoplakin (red) was used to identify cardiomyocyte and Hoechst was used to detect nuclei(right). Bar $=100 \mu \mathrm{m}$. b. Variable region and constant region of Igk were amplified by RT-PCR in WT and $\mu \mathrm{MT}$ mice heart tissue. c. The VJ recombination pattern in cardiomyocyte of WT and $\mu \mathrm{MT}$ mice. d. Sequence of Igk V12-46/J2 and IgVK 
14-111/J5, which is the dominant V-J recombination pattern in isolated heart cells. (B) Sequence analysis of Igk variable region in cardiomyocyte and fibroblast of neonatal mice. (C) Sequence analysis of Igk variable region in $\mathrm{HL}-1$ cells. (D) a. Igk transcript was detected in single cardiomyocyte of WT mice by RTPCR. Myh6, cTnl, cTnT was used as cardiomyocyte marker and heart tissue of WT mice was used as positive control. CD20 was used as B cell marker and spleen of WT mice was used as positive control. b. Igk transcript was detected in single cardiomyocyte of $\mu \mathrm{MT}$ mice by RT-PCR. c. V segment used in single cardiomyocyte $(n=42), 36$ of WT single cardiomyocytes (No.1-36) and 6 of $\mu$ MT single cardiomyocytes (Np.37-42) were analyzed. d. V region usage of 20 single cardiomyocytes including 17 WT cardiomyocytes (No.1-17) and $3 \mu \mathrm{MT}$ cardiomyocytes (No.18-20) were analyzed by Sanger sequencing. e. $V$ region usage of the single cardiomyocyte mentioned in Fig2Dd were testified by using the next generation sequencing. $f$. Proportion of different number functional $V$ region usage in a single cardiomyocyte was analyzed $(n=42)$ derived from WT $(n=36)$ and $\mu$ MT mice $(n=6)$ by Sanger sequencing (left) and 20 of the single cardiomyocytes were testified by the next generation sequencing. g. Proportion of different number functional $V_{k} J_{k}$ recombination pattern in a single cardiomyocyte was analyzed( $n=42)$ by Sanger sequencing. $h$. The frequency of $V_{\kappa}$ gene segments used by cardiomyocyte compared with B cells by Sanger sequencing and testified by the next generation sequencing. $i$. The frequency of $\mathrm{J} k$ gene segments used by cardiomyocyte compared with B cells by Sanger sequencing. $\mathrm{j}$. Sequence of Igk variable region in single cardiomyocyte. 


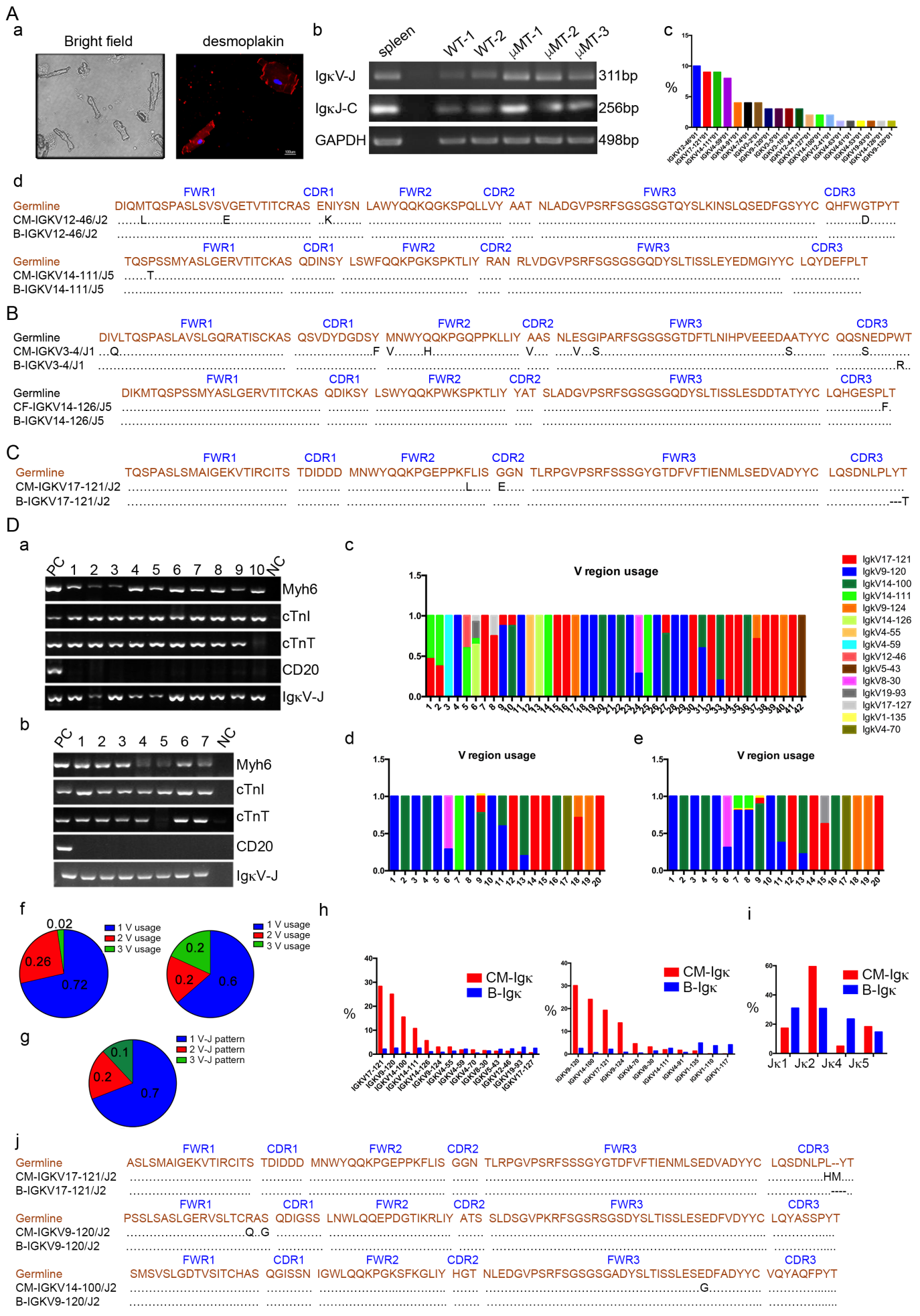

Figure 2

Igk transcripts were expressed in cardiomyocyte with restricted diversity compared with B cell (A) a. Isolated cardiomyocyte of adult mice under the bright field of microscopy(left). Desmoplakin (red) was used to identify cardiomyocyte and Hoechst was used to detect nuclei(right). Bar $=100 \mu \mathrm{m}$. b. Variable region and constant region of Igk were amplified by RT-PCR in WT and $\mu \mathrm{MT}$ mice heart tissue. c. The VJ recombination pattern in cardiomyocyte of WT and $\mu \mathrm{MT}$ mice. d. Sequence of Igk V12-46/J2 and IgVK 
14-111/J5, which is the dominant V-J recombination pattern in isolated heart cells. (B) Sequence analysis of Igk variable region in cardiomyocyte and fibroblast of neonatal mice. (C) Sequence analysis of Igk variable region in $\mathrm{HL}-1$ cells. (D) a. Igk transcript was detected in single cardiomyocyte of WT mice by RTPCR. Myh6, cTnl, cTnT was used as cardiomyocyte marker and heart tissue of WT mice was used as positive control. CD20 was used as B cell marker and spleen of WT mice was used as positive control. b. Igk transcript was detected in single cardiomyocyte of $\mu \mathrm{MT}$ mice by RT-PCR. c. V segment used in single cardiomyocyte $(n=42), 36$ of WT single cardiomyocytes (No.1-36) and 6 of $\mu$ MT single cardiomyocytes (Np.37-42) were analyzed. d. V region usage of 20 single cardiomyocytes including 17 WT cardiomyocytes (No.1-17) and $3 \mu \mathrm{MT}$ cardiomyocytes (No.18-20) were analyzed by Sanger sequencing. e. $V$ region usage of the single cardiomyocyte mentioned in Fig2Dd were testified by using the next generation sequencing. $f$. Proportion of different number functional $V$ region usage in a single cardiomyocyte was analyzed $(n=42)$ derived from WT $(n=36)$ and $\mu$ MT mice $(n=6)$ by Sanger sequencing (left) and 20 of the single cardiomyocytes were testified by the next generation sequencing. g. Proportion of different number functional $V_{k} J_{k}$ recombination pattern in a single cardiomyocyte was analyzed( $n=42)$ by Sanger sequencing. $h$. The frequency of $V_{\kappa}$ gene segments used by cardiomyocyte compared with B cells by Sanger sequencing and testified by the next generation sequencing. $i$. The frequency of $\mathrm{J} k$ gene segments used by cardiomyocyte compared with B cells by Sanger sequencing. $\mathrm{j}$. Sequence of Igk variable region in single cardiomyocyte. 

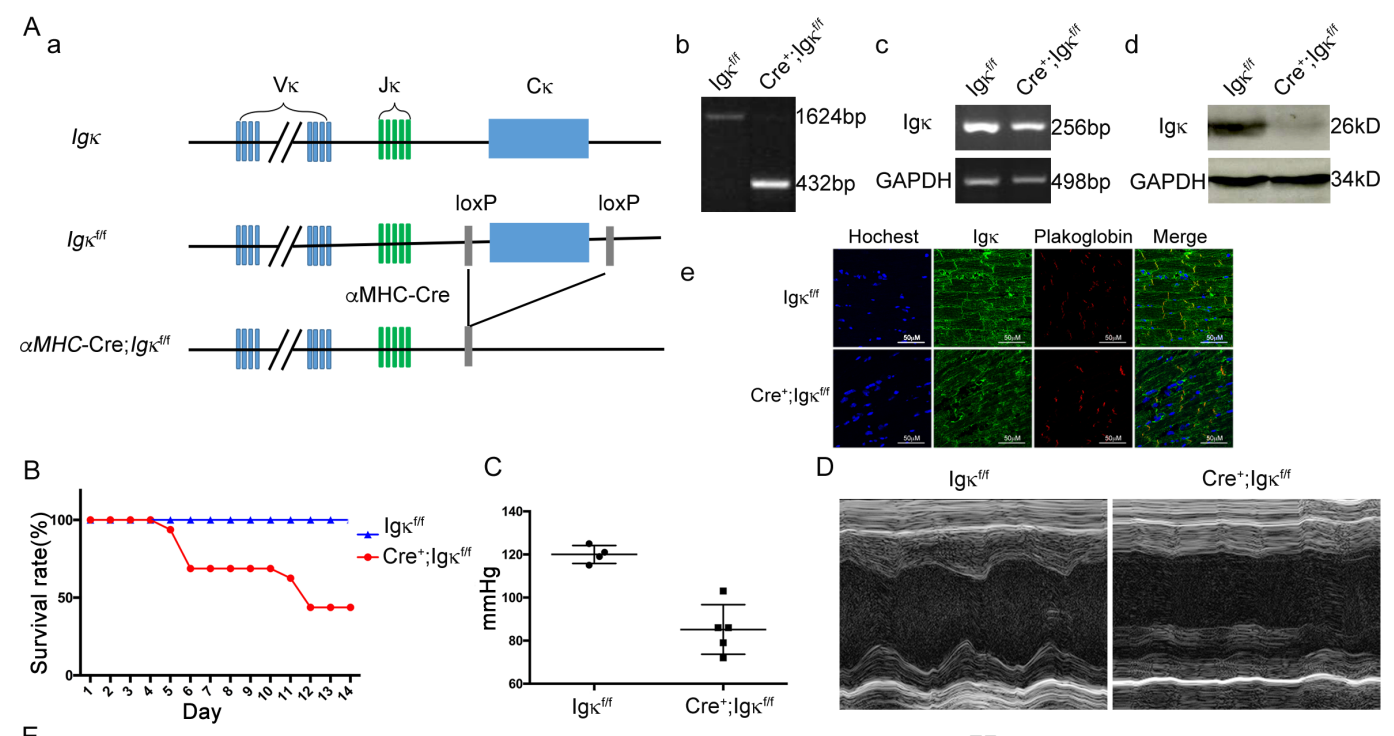

C
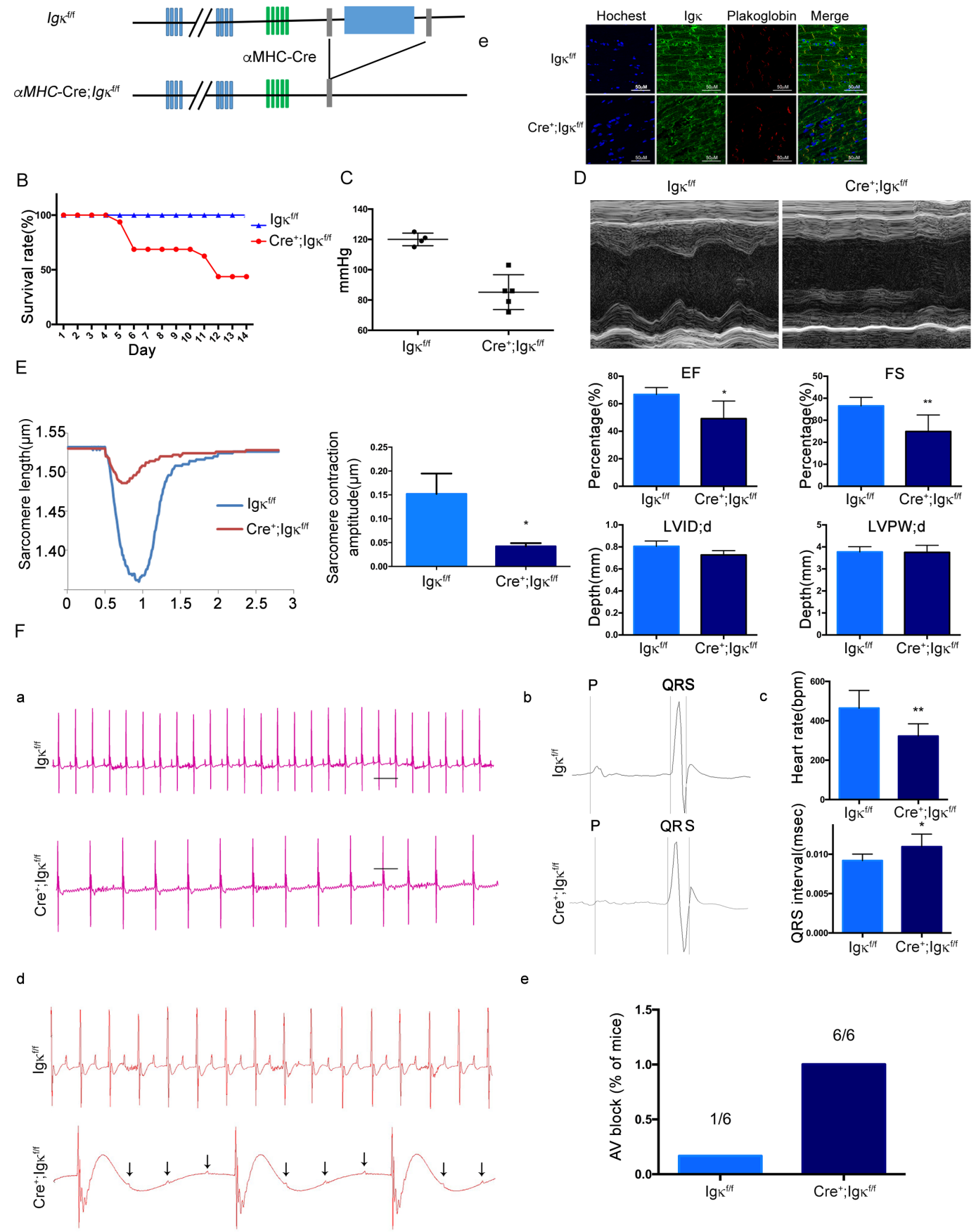

Figure 3

IgK cKO mice display myocardial contraction dysfunction and conduction defects. (A) a. Scheme for generation of cardiac conditional Igk knock out mouse (cKO). LoxP sites were inserted to flank exons in Igk gene constant region. Mice homozygous for loxP insertion ( $f / f)$ were crossed with mice expressing Cre recombinase under control of the $\mu \mathrm{MHC}$ promoter. Cre-mediated excision of Igk constant region resulted in defect of Igk gene structure and dysfunction. b. Genotype of control and cKO mice were 
identified by PCR. Primers were designed on both sides of flox region, the product size was 432bp when Igk gene was deleted. c. Igk expression on mRNA level in control and cKO mouse heart tissue by RT-PCR. d. Igk expression on protein level in control and cKO mouse heart tissue by Western blot. e. Colocalization of IgK (green) and Plakoglobin (red) on the ICD in control and CKO heart tissue. Hoechst (blue) was used

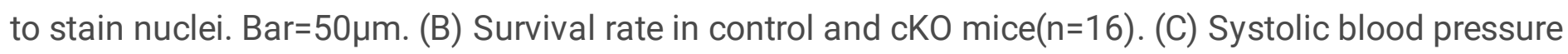
$(S B P)$ in control $(n=4)$ and $c K O$ mice $(n=5)$. (D) Echocardiographic assessment of heart function using Mmode images of left ventricle(left), and quantification of left ventricular posterior wall thicknesses (LVPW), left ventricular inner dimension (LVID), left ventricular fractional shortening (FS) and the ejection fraction (EF) showed contraction dysfunction in cKO mice $(n=6)$. (E) Individual traces of sarcomere shortening in isolated ventricular myocytes of control and cKO mice at $0.5 \mathrm{~Hz}$. Six twitches per myocyte were collected for each sample ( $n=2)$. (F) IgK cKO mice display bradycardia compared with control mice (control: $n=5$; cKO: $n=7 ; p<0.05$ ). Bar $=200$ ms. a. Representative ECG traces for control and cKO mice. b,c. cKO mice show increased QRS intervals compared with control mice (WT: $n=6 ; c K O: n=7 ; p<0.05)$. $d$. ECG traces from control and cKO mouse 30 seconds prior to ISO administration. ECG traces from same mice observed 10 minutes post-ISO administration ( $20 \mathrm{mg} / \mathrm{kg}$, I.P.; bar=200 ms). e. Examples of AV block (arrowheads) are noted in cKO mice show significant decrease in heart rate and increase in frequency of AV block following ISO compared with control mice. 

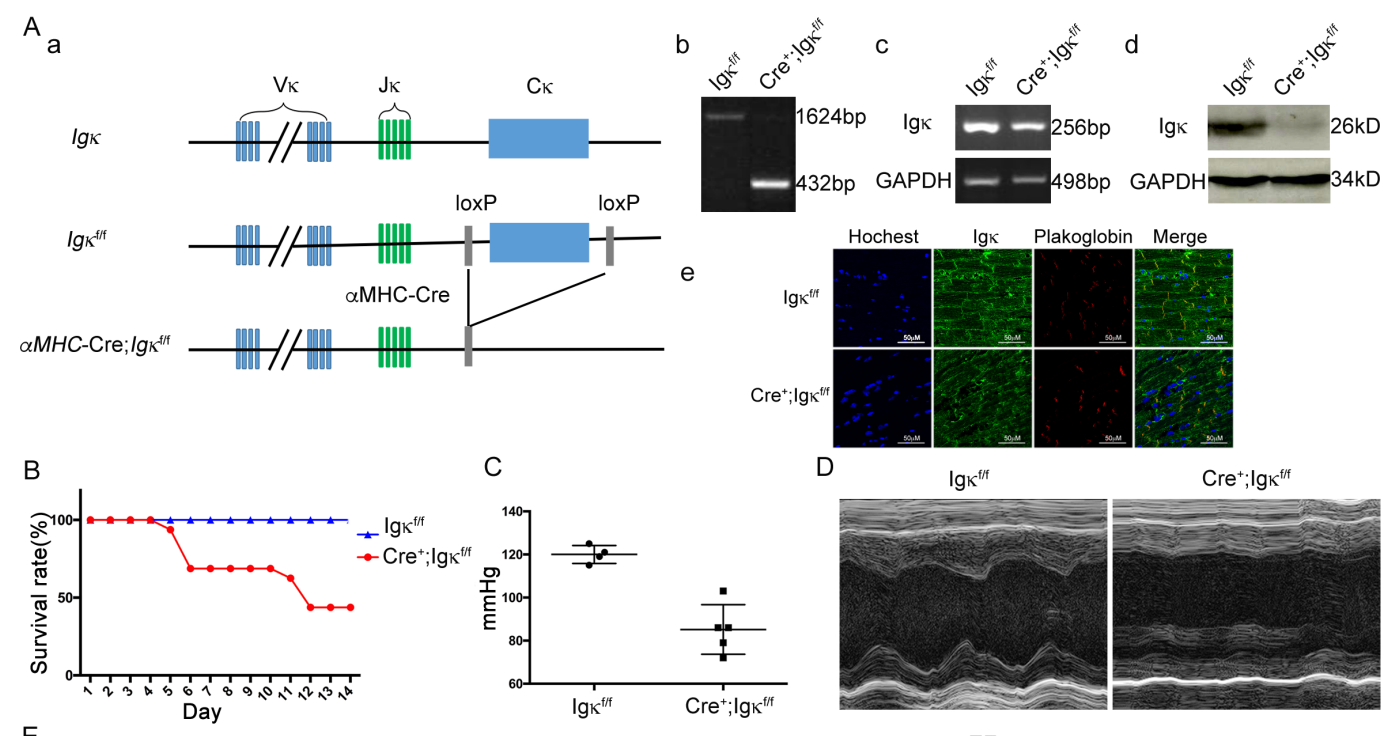

C
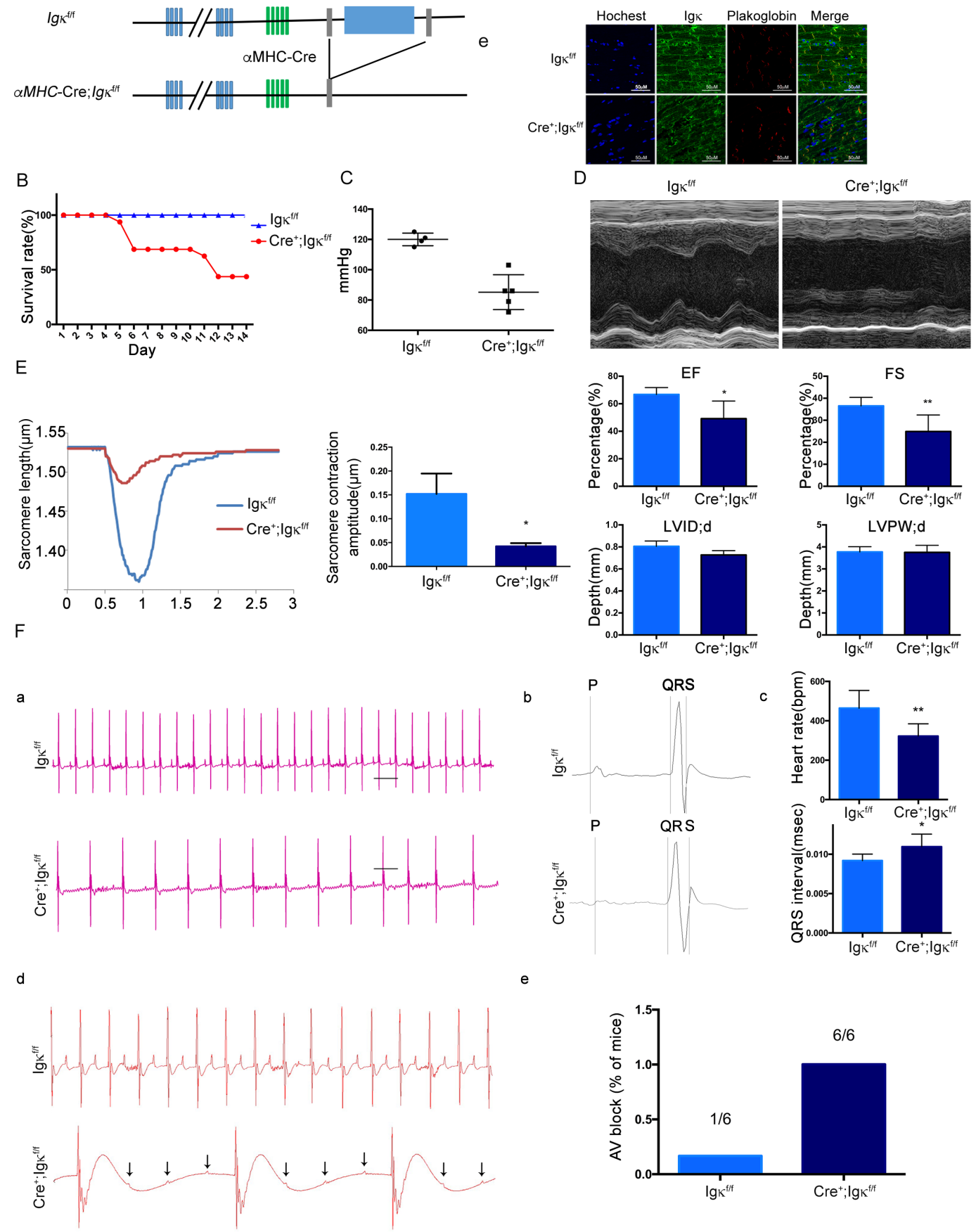

Figure 3

IgK cKO mice display myocardial contraction dysfunction and conduction defects. (A) a. Scheme for generation of cardiac conditional Igk knock out mouse (cKO). LoxP sites were inserted to flank exons in Igk gene constant region. Mice homozygous for loxP insertion ( $f / f)$ were crossed with mice expressing Cre recombinase under control of the $\mu \mathrm{MHC}$ promoter. Cre-mediated excision of Igk constant region resulted in defect of Igk gene structure and dysfunction. b. Genotype of control and cKO mice were 
identified by PCR. Primers were designed on both sides of flox region, the product size was 432bp when Igk gene was deleted. c. Igk expression on mRNA level in control and cKO mouse heart tissue by RT-PCR. d. Igk expression on protein level in control and cKO mouse heart tissue by Western blot. e. Colocalization of Igk (green) and Plakoglobin (red) on the ICD in control and cKO heart tissue. Hoechst (blue) was used

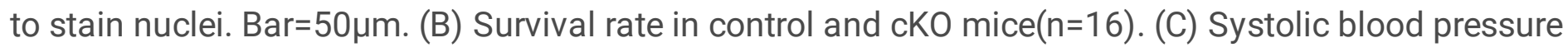
$(S B P)$ in control $(n=4)$ and $c K O$ mice $(n=5)$. (D) Echocardiographic assessment of heart function using Mmode images of left ventricle(left), and quantification of left ventricular posterior wall thicknesses (LVPW), left ventricular inner dimension (LVID), left ventricular fractional shortening (FS) and the ejection fraction (EF) showed contraction dysfunction in cKO mice $(n=6)$. (E) Individual traces of sarcomere shortening in isolated ventricular myocytes of control and cKO mice at $0.5 \mathrm{~Hz}$. Six twitches per myocyte were collected for each sample ( $n=2)$. (F) IgK cKO mice display bradycardia compared with control mice (control: $n=5$; cKO: $n=7 ; p<0.05$ ). Bar $=200$ ms. a. Representative ECG traces for control and cKO mice. b,c. cKO mice show increased QRS intervals compared with control mice (WT: $n=6 ; c K O: n=7 ; p<0.05)$. $d$. ECG traces from control and CKO mouse 30 seconds prior to ISO administration. ECG traces from same mice observed 10 minutes post-ISO administration ( 20 mg/kg, I.P.; bar=200 ms). e. Examples of AV block (arrowheads) are noted in cKO mice show significant decrease in heart rate and increase in frequency of AV block following ISO compared with control mice.

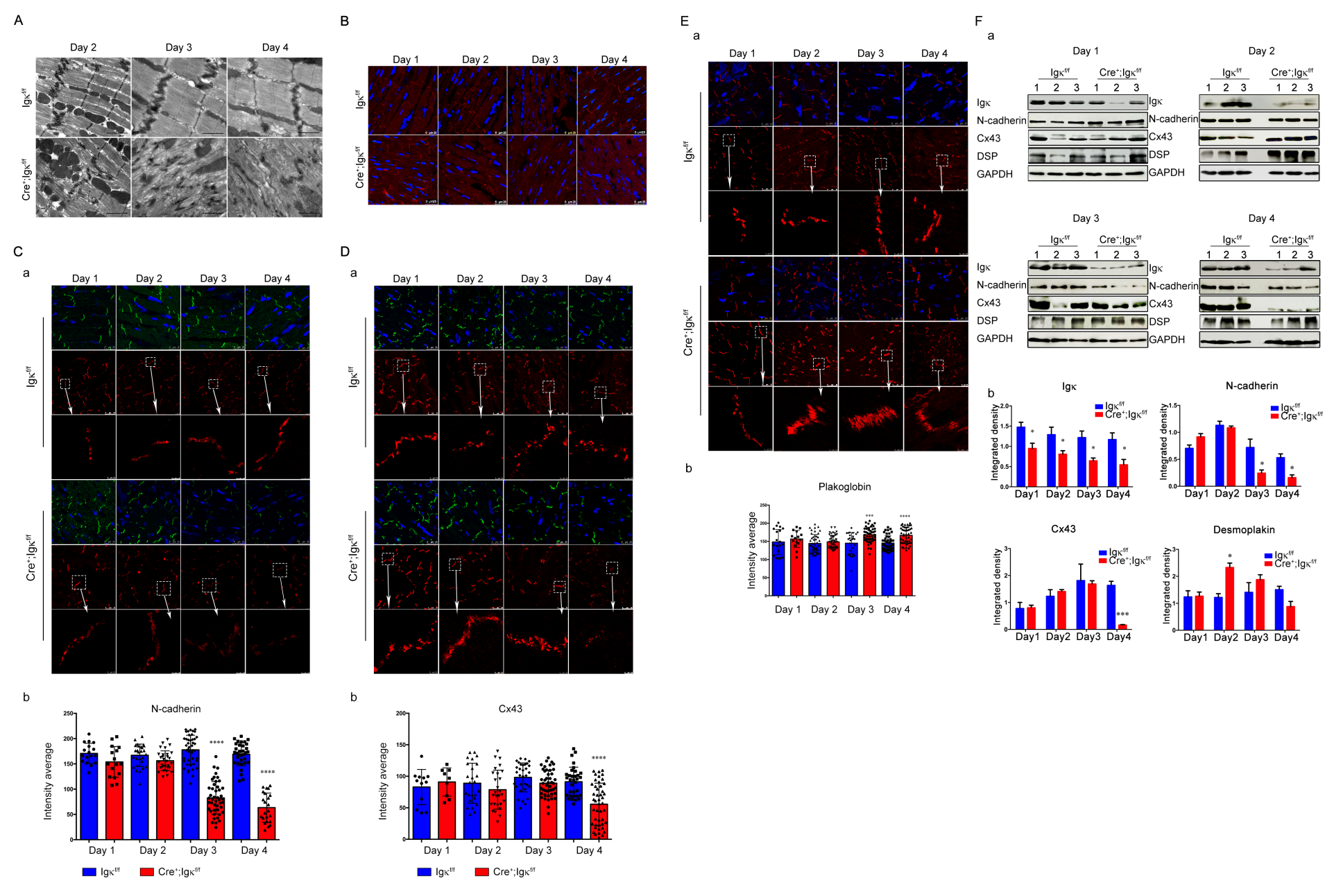

Figure 4 
Igк cKO mice display abnormal structure of intercalated disc and defects in $\mathrm{Cx} 43$ and $\mathrm{N}$-cadherin expression. (A) The structure of ICD in the heart tissue of control and CKO mice by transmission electron microscopy. cKO mice shows severe destruction of ICD structure. Bar $=0.5 \mu \mathrm{m}$. (B) IgK staining(red) in both control and cKO mice for consecutive five days after tamoxifen injection. Ig staining was decreased on

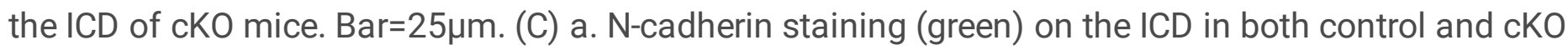
mice for consecutive four days after tamoxifen injection by confocal (green) and STED (red). b. The average intensity of the $\mathrm{N}$-cadherin staining. The staining of $\mathrm{N}$-cadherin was reduced at day 3 and day 4 after tamoxifen administration. (D) a. Cx43 staining (green) on the ICD in both control and cKO mice for consecutive four days after tamoxifen injection by confocal (green) and STED (red). b. The average intensity of the $\mathrm{Cx} 43$ staining. The staining of $\mathrm{Cx} 43$ was reduced at day 3 and day 4 after tamoxifen administration. (E) a. Plakoglobin staining (red) on the ICD in both control and cKO mice for consecutive four days after tamoxifen injection by confocal (red) and STED (red). b. The average intensity of the plakoglobin staining. The staining of plakoglobin was increased at day 3 and day 4 after tamoxifen administration. (F) a. Expression of IgK, desmoplakin, $\mathrm{Cx} 43$ and $\mathrm{N}$-cadherin on protein level of control and CKO mice for consecutive five days after tamoxifen injection by Western blot. GAPDH was used as an internal control. (D) IgK, Desmoplakin, $\mathrm{Cx} 43$ and $\mathrm{N}$-cadherin on protein level in Fig 4Fa are normalized to GAPDH loading control $(\mathrm{p}<0.05)$.

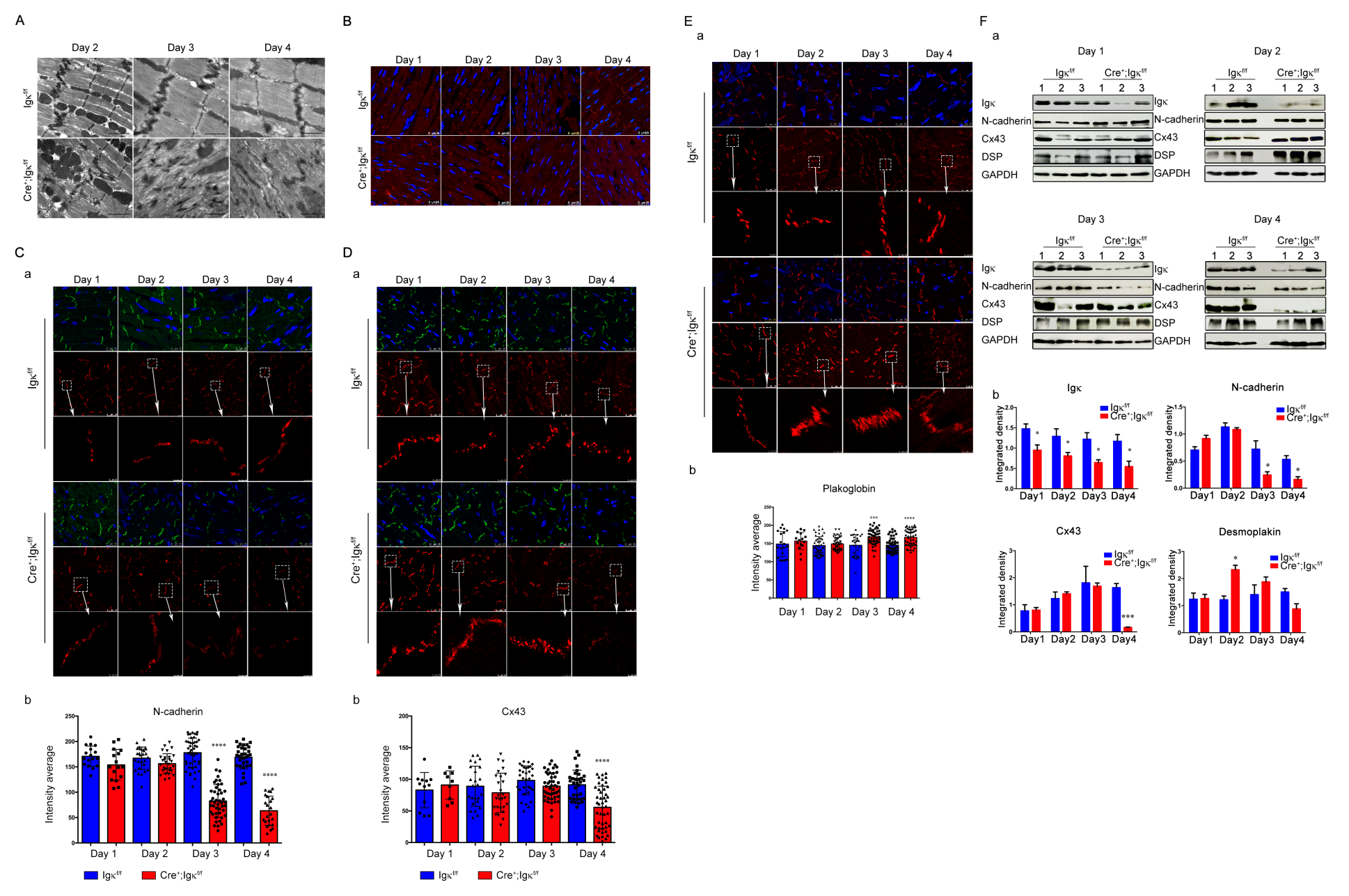

Figure 4 
Igk cKO mice display abnormal structure of intercalated disc and defects in $\mathrm{Cx} 43$ and $\mathrm{N}$-cadherin expression. (A) The structure of ICD in the heart tissue of control and CKO mice by transmission electron microscopy. CKO mice shows severe destruction of ICD structure. Bar $=0.5 \mu \mathrm{m}$. (B) IgK staining(red) in both control and CKO mice for consecutive five days after tamoxifen injection. Ig staining was decreased on

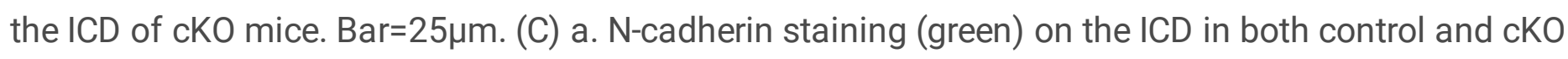
mice for consecutive four days after tamoxifen injection by confocal (green) and STED (red). b. The average intensity of the $\mathrm{N}$-cadherin staining. The staining of $\mathrm{N}$-cadherin was reduced at day 3 and day 4 after tamoxifen administration. (D) a. CX43 staining (green) on the ICD in both control and cKO mice for consecutive four days after tamoxifen injection by confocal (green) and STED (red). b. The average intensity of the $\mathrm{Cx} 43$ staining. The staining of $\mathrm{Cx} 43$ was reduced at day 3 and day 4 after tamoxifen administration. (E) a. Plakoglobin staining (red) on the ICD in both control and cKO mice for consecutive four days after tamoxifen injection by confocal (red) and STED (red). b. The average intensity of the plakoglobin staining. The staining of plakoglobin was increased at day 3 and day 4 after tamoxifen administration. (F) a. Expression of Igk, desmoplakin, $\mathrm{Cx} 43$ and $\mathrm{N}$-cadherin on protein level of control and cKO mice for consecutive five days after tamoxifen injection by Western blot. GAPDH was used as an internal control. (D) Igk, Desmoplakin, Cx43 and N-cadherin on protein level in Fig 4Fa are normalized to GAPDH loading control $(p<0.05)$. 


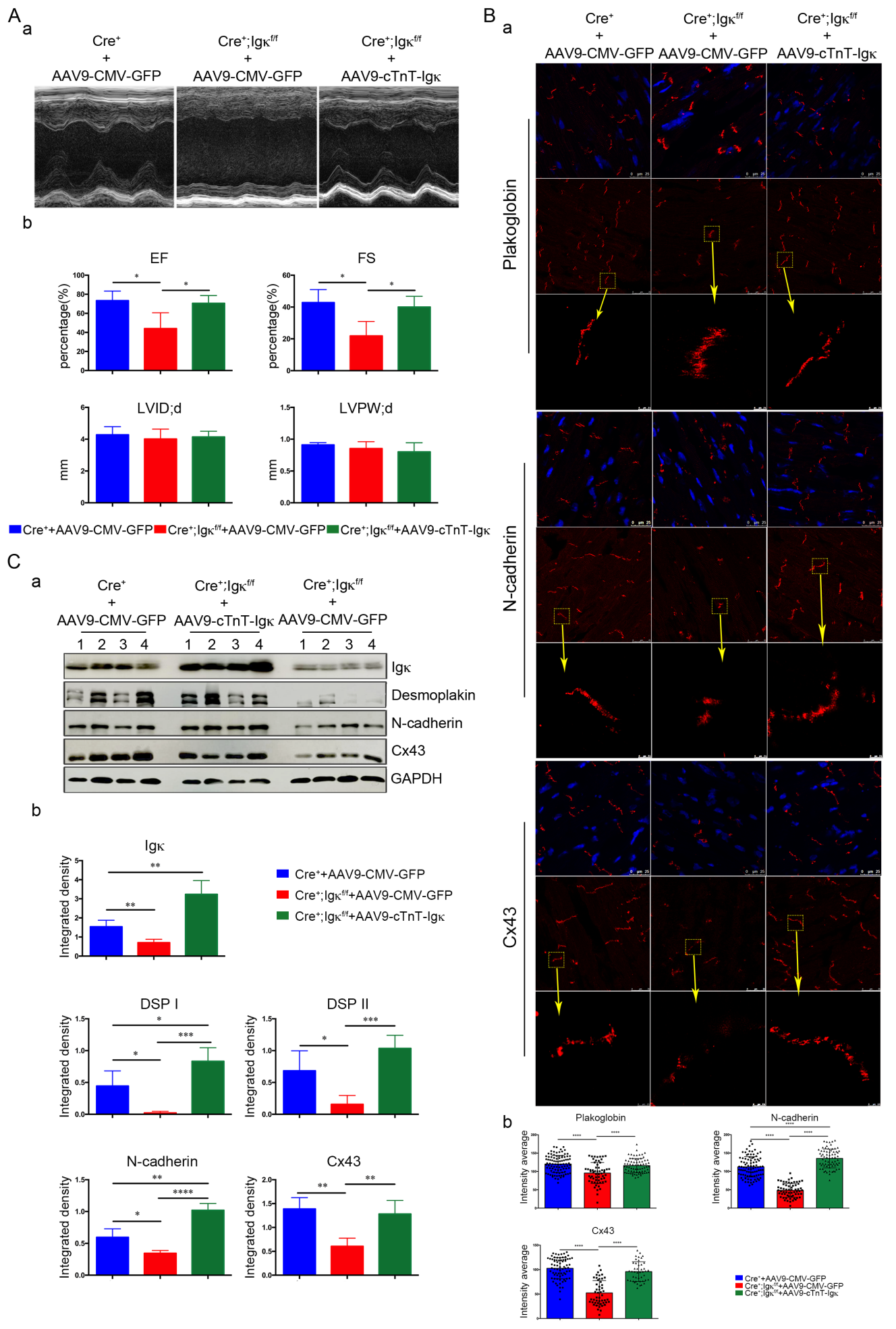

\section{Figure 5}

Dysfunction of cardiomyocyte and abnormal structure of ICD caused by Igk deficiency can be rescued by overexpression of Igk using AAV9-cTnT-Ig Igk vector. (A) a. Echocardiographic assessment of heart function in cKO mice, control mice and cKO mice injected with AAV9-cTnT-Ig Igk vector $(n=4)$. b. Evaluation of cardiac constriction function by EF and FS shows that the cardiac function was improved by overexpressing Igk in Igk cKO mice. (B) a. The staining of plakoglobin, $\mathrm{N}$-cadherin and Cx43 in cKO 
mice, control mice and cKO mice injected with AAV9-cTnT-Ig Igk vector. Injected with AAV9-cTnT-Ig IgK vector in CKO mice showed improved ICD structure and increased the expression of $\mathrm{N}$-cadherin and $\mathrm{Cx} 43$ on the ICD. b. The average intensity of the plakoglobin, $\mathrm{N}$-cadherin and $\mathrm{Cx} 43$ staining. (C) a. The protein level of desmoplakin, $\mathrm{N}$-cadherin and $\mathrm{Cx} 43$ in cKO mice, control mice and cKO mice injected with AAV9cTnT-Ig Igk vector by Western blot. b. After normalized to GAPDH loading control, the protein level of Desmoplakin, $\mathrm{N}$-cadherin and $\mathrm{C} \times 43$ were upregulated by Igk overexpression in IgK cKO mice.

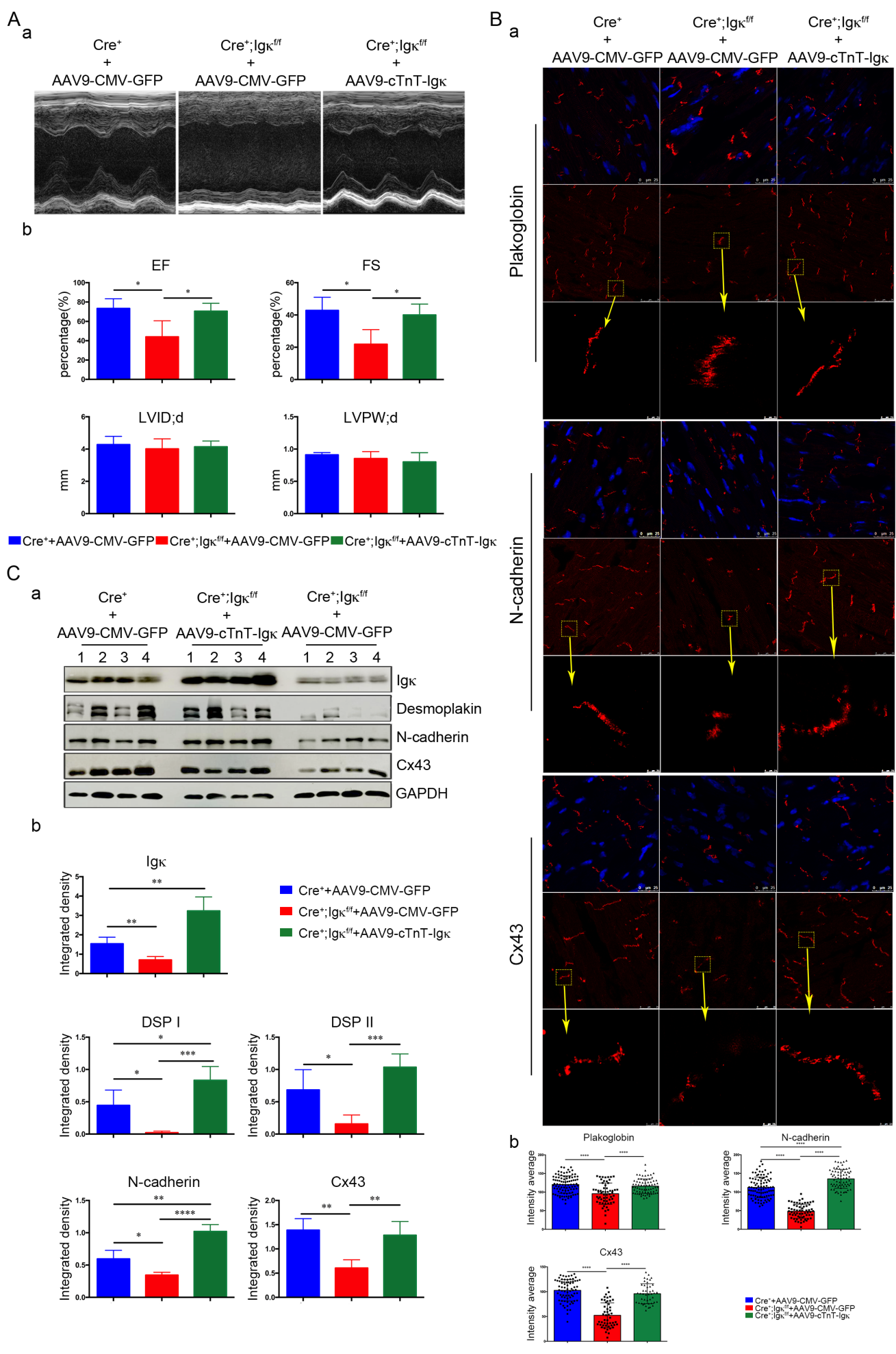

Figure 5 
Dysfunction of cardiomyocyte and abnormal structure of ICD caused by Igk deficiency can be rescued by overexpression of Igk using AAV9-cTnT-Ig Igk vector. (A) a. Echocardiographic assessment of heart function in cKO mice, control mice and cKO mice injected with AAV9-cTnT-Ig IgK vector $(n=4)$. b.

Evaluation of cardiac constriction function by EF and FS shows that the cardiac function was improved by overexpressing Igk in IgK CKO mice. (B) a. The staining of plakoglobin, $\mathrm{N}$-cadherin and $\mathrm{Cx} 43$ in $\mathrm{cKO}$ mice, control mice and cKO mice injected with AAV9-cTnT-Ig Igk vector. Injected with AAV9-cTnT-Ig IgK vector in cKO mice showed improved ICD structure and increased the expression of $\mathrm{N}$-cadherin and $\mathrm{Cx} 43$ on the ICD. b. The average intensity of the plakoglobin, $\mathrm{N}$-cadherin and $\mathrm{Cx} 43$ staining. (C) a. The protein level of desmoplakin, N-cadherin and $\mathrm{Cx} 43$ in cKO mice, control mice and cKO mice injected with AAV9cTnT-Ig Igk vector by Western blot. b. After normalized to GAPDH loading control, the protein level of Desmoplakin, $\mathrm{N}$-cadherin and $\mathrm{Cx} 43$ were upregulated by Igк overexpression in IgK cKO mice. 
A

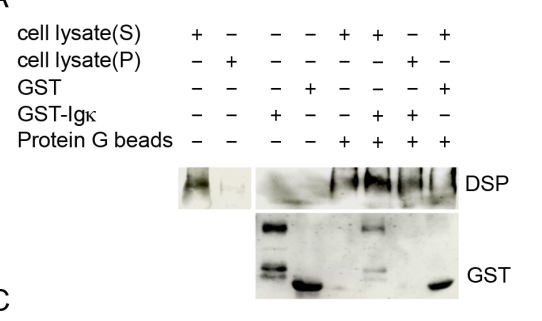

C

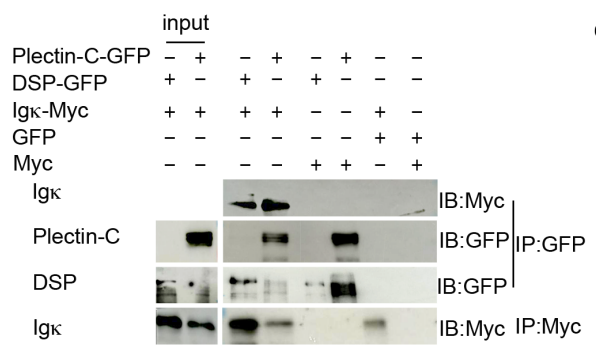

$\mathrm{D}$
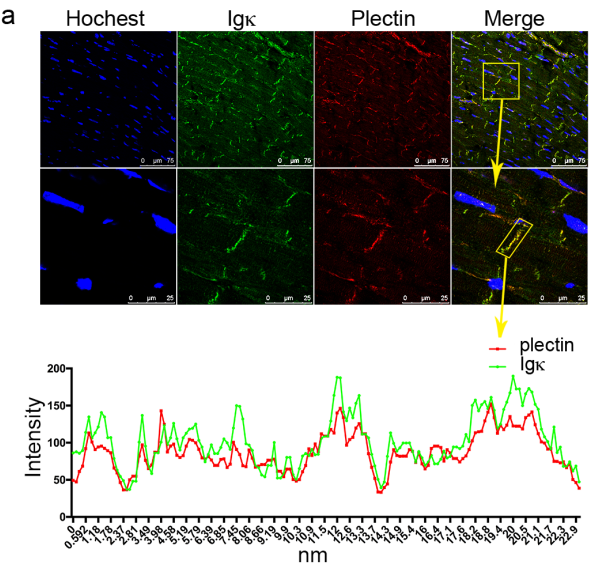

b

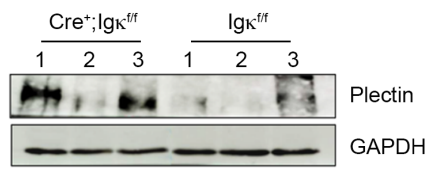

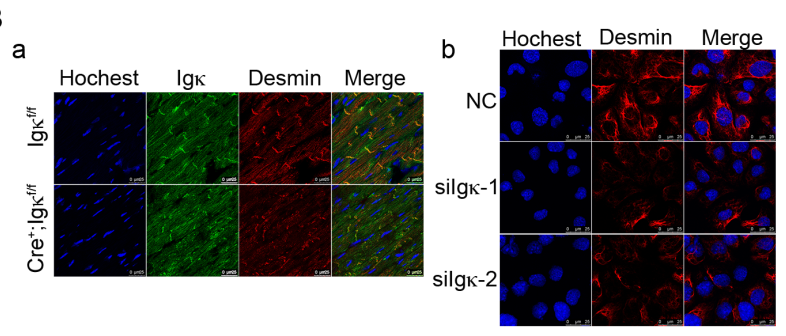
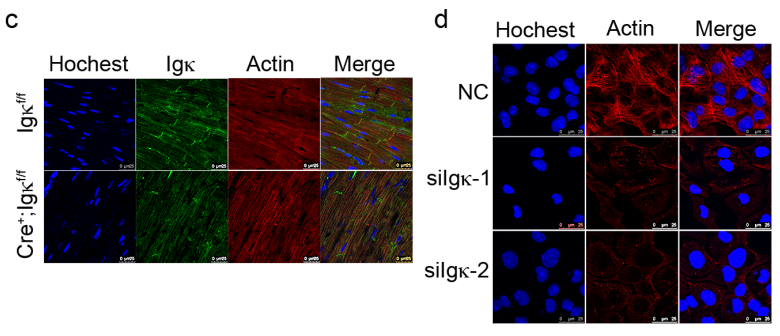
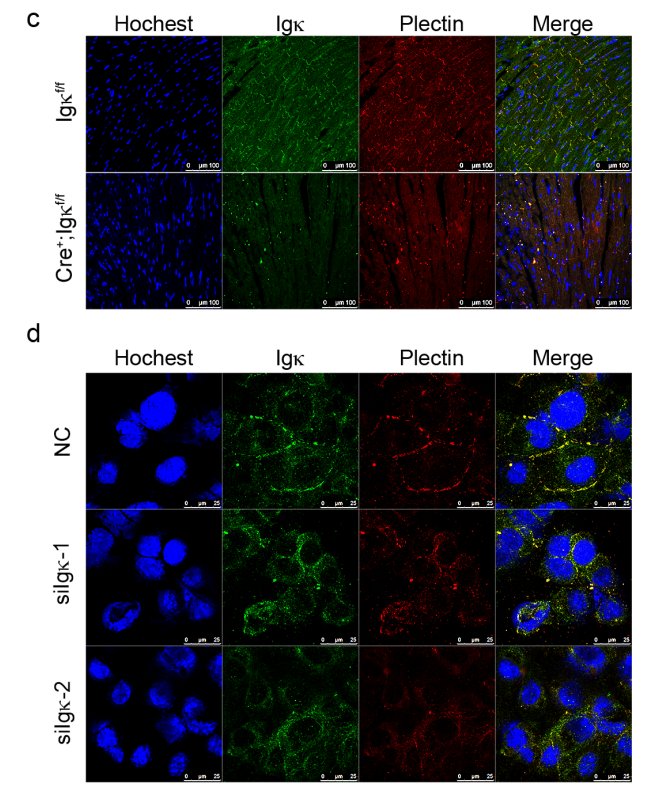

$\mathrm{E}$

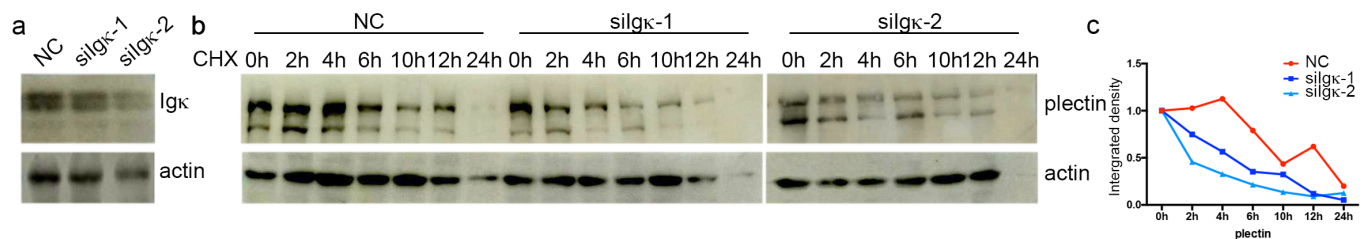

\section{Figure 6}

CM-Igk can interact with Plectin, reduction of CM-Igk induces degradation of plectin and loss of location on the ICD. (A) Results of GST pull down experiment by using GST-IgK/GST and heart tissue lysate. Western blot was used to identify desmoplakin and GST. (B) plectin-C-GFP/DSP-GFP and Igk-Myc were co-transfected into 293T cells and total cell lysate was extracted and subjected to IP assay using GFP antibody. Western blot was used to detect Igk, plectin-C and DSP by using anti-Myc, anti-GFP antibody. 
(C) a. Colocalization of Igk and plectin on the ICD by immunofluorescence assay. The colocalization rate is 0.7781 b. Expression of plectin in the heart tissue of IgK cKO and control mice by Western blot assay. c. Immunofluorescence staining for IgK and plectin on the ICD of IgK cKO and control mice. d. Immunofluorescence staining for Igk and plectin on the cell junction of HL-1 cells. Two specific siRNA for IgK was used to knock down Igk in HL-1 cells. (D) a. The expression of IgK was decreased in HL-1 cells by using two specific siRNA for Igk. b. Igk knocked down resulted in a rapid degradation of plectin protein in $\mathrm{HL}-1$ cells. Cells were co-treated with cycloheximide (CHX), and harvested at $0,2,4,6,10,12$ and $24 \mathrm{~h}$ after the treatments for western blotting analysis. c. Plectin on protein level in Fig $6 \mathrm{Db}$ are normalized to GAPDH loading control $(p<0.05)$. (E) a. Immunofluorescence staining for Igk and desmin on the ICD of Igk cKO and control mice. b. Immunofluorescence staining for lgk and desmin in HL-1 cells. Two specific siRNA for Igk was used to knock down Igk in HL-1 cells. 
A

cell lysate(S) $\quad+-\quad-++-+$

cell lysate $(\mathrm{P}) \quad-+--{ }_{-}+-$

GST $\quad-\quad-+\cdots+-$

GST-IgK

Protein $\mathrm{G}$ beads

it.

C

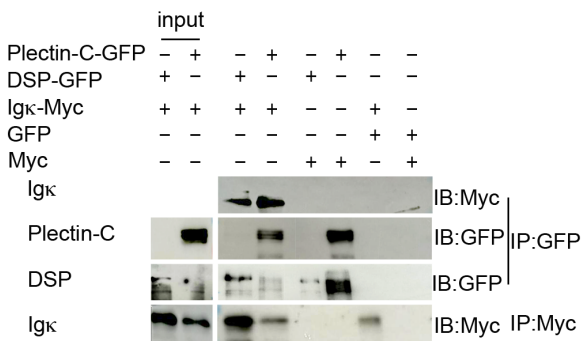

$\mathrm{D}$
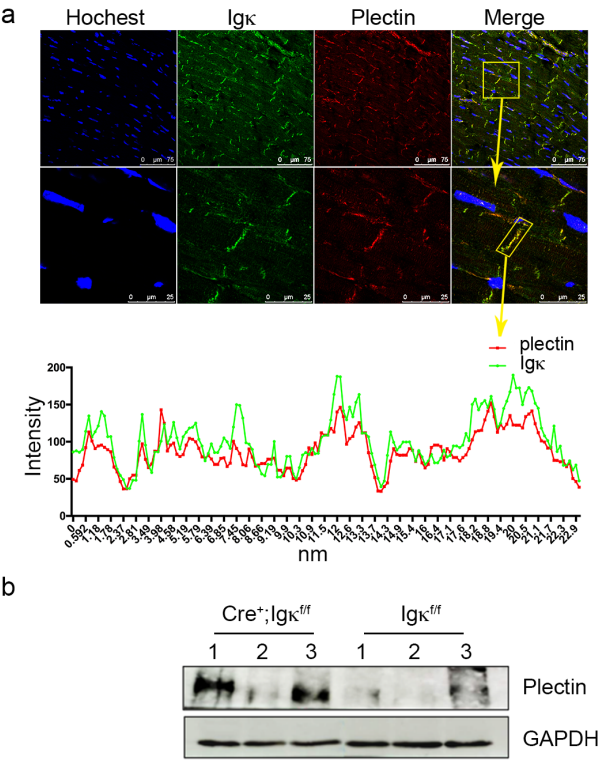

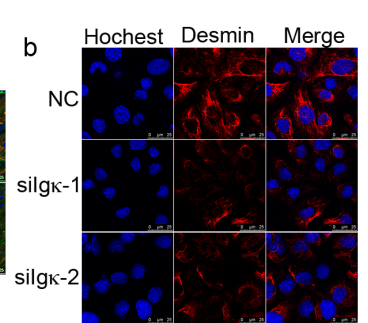

d Hochest Actin Merge

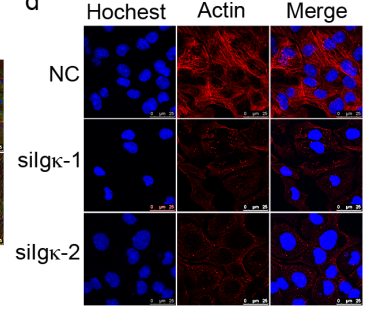

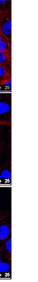
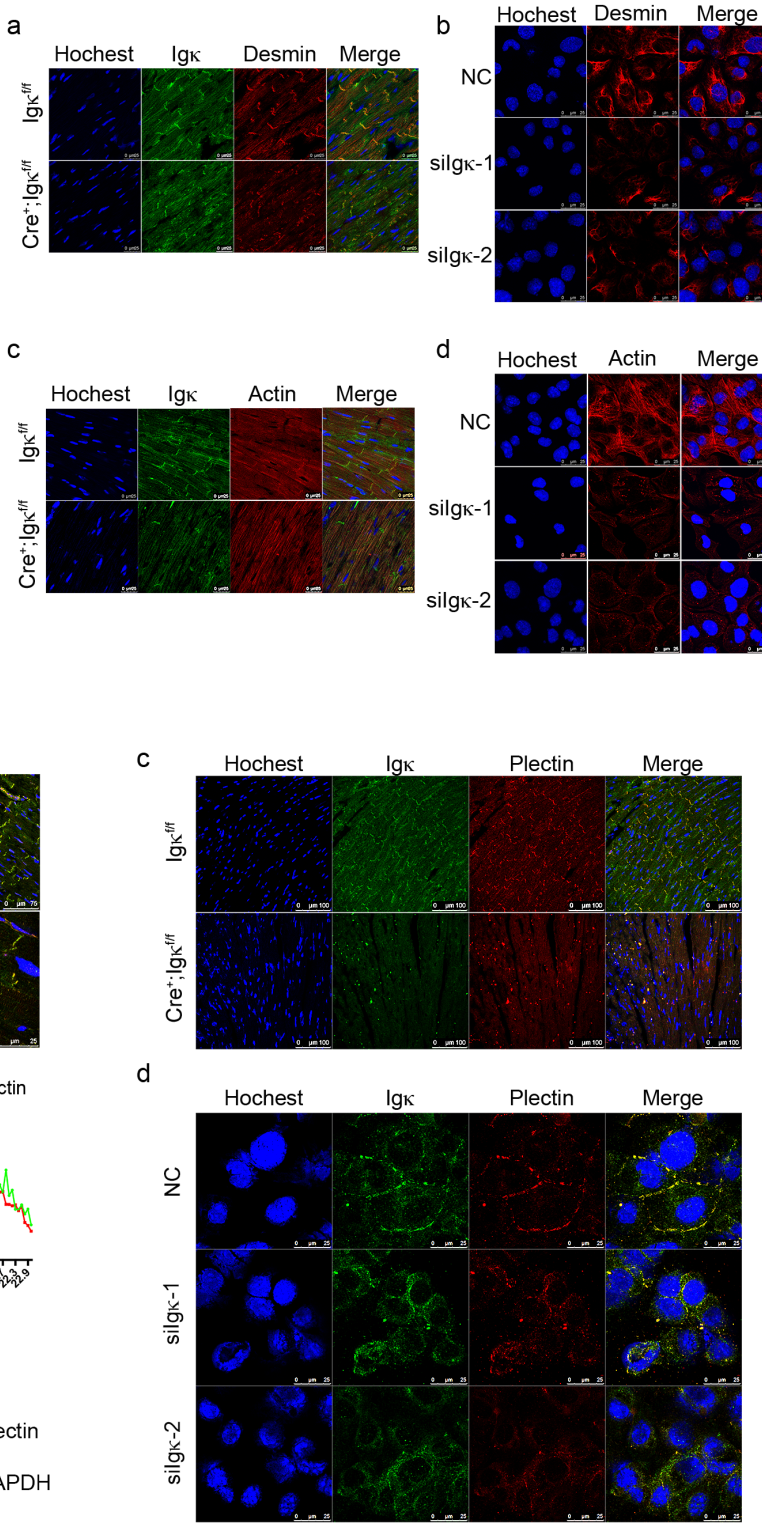

E

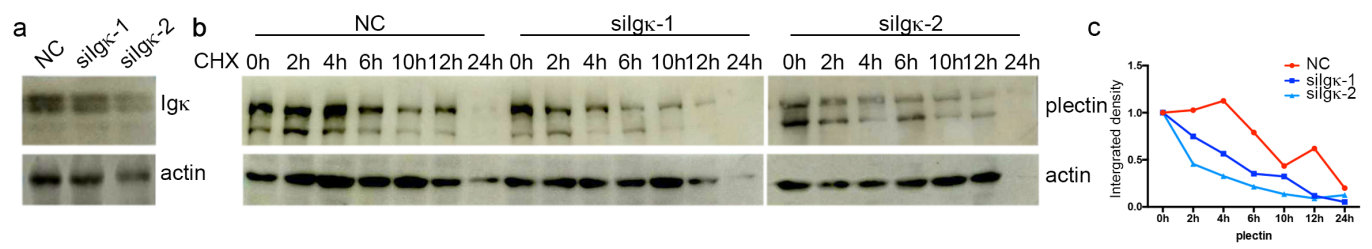

\section{Figure 6}

CM-Igk can interact with Plectin, reduction of CM-Igk induces degradation of plectin and loss of location on the ICD. (A) Results of GST pull down experiment by using GST-IgK/GST and heart tissue lysate. Western blot was used to identify desmoplakin and GST. (B) plectin-C-GFP/DSP-GFP and Igk-Myc were co-transfected into 293T cells and total cell lysate was extracted and subjected to IP assay using GFP antibody. Western blot was used to detect Igk, plectin-C and DSP by using anti-Myc, anti-GFP antibody. 
(C) a. Colocalization of Igk and plectin on the ICD by immunofluorescence assay. The colocalization rate is 0.7781 . b. Expression of plectin in the heart tissue of Igk CKO and control mice by Western blot assay. c. Immunofluorescence staining for IgK and plectin on the ICD of IgK cKO and control mice. d. Immunofluorescence staining for Igk and plectin on the cell junction of HL-1 cells. Two specific siRNA for Igk was used to knock down Igk in HL-1 cells. (D) a. The expression of Igk was decreased in HL-1 cells by using two specific siRNA for Igk. b. Igk knocked down resulted in a rapid degradation of plectin protein in $\mathrm{HL}-1$ cells. Cells were co-treated with cycloheximide (CHX), and harvested at $0,2,4,6,10,12$ and $24 \mathrm{~h}$ after the treatments for western blotting analysis. c. Plectin on protein level in Fig 6Db are normalized to GAPDH loading control $(p<0.05)$. (E) a. Immunofluorescence staining for IgK and desmin on the ICD of

Igk cKO and control mice. b. Immunofluorescence staining for lgk and desmin in HL-1 cells. Two specific siRNA for Igk was used to knock down Igk in HL-1 cells.

\section{Supplementary Files}

This is a list of supplementary files associated with this preprint. Click to download.

- FigS1.tif

- Figs1.tif

- Figs2.tif

- Figs2.tif

- FigS3.tif

- Figs3.tif

- supplymentaryTable1.docx

- supplymentaryTable1.docx

- supplementarytable2.xlsx

- supplementarytable2.xlsx

- supplementaryTable3.docx

- supplementaryTable3.docx

- supplymentarytable4.xlsx

- supplymentarytable4.xlsx 